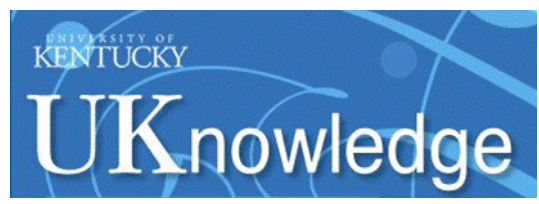

University of Kentucky

UKnowledge

\title{
Determination of the Physical Conditions of the Knots in the Helix Nebula from Optical and Infrared Observations
}

\author{
C. R. O'Dell \\ Vanderbilt University
}

W. J. Henney

Universidad Nacional Autónoma de México, Mexico

Gary J. Ferland

University of Kentucky, gary@uky.edu

Follow this and additional works at: https://uknowledge.uky.edu/physastron_facpub

Part of the Astrophysics and Astronomy Commons, and the Physics Commons

Right click to open a feedback form in a new tab to let us know how this document benefits you.

\section{Repository Citation}

O'Dell, C. R.; Henney, W. J.; and Ferland, Gary J., "Determination of the Physical Conditions of the Knots in the Helix Nebula from Optical and Infrared Observations" (2007). Physics and Astronomy Faculty Publications. 133.

https://uknowledge.uky.edu/physastron_facpub/133

This Article is brought to you for free and open access by the Physics and Astronomy at UKnowledge. It has been accepted for inclusion in Physics and Astronomy Faculty Publications by an authorized administrator of UKnowledge. For more information, please contact UKnowledge@lsv.uky.edu. 
Determination of the Physical Conditions of the Knots in the Helix Nebula from Optical and Infrared Observations

\author{
Digital Object Identifier (DOI) \\ http://dx.doi.org/10.1086/513011 \\ Notes/Citation Information \\ Published in The Astronomical Journal, v. 113, no. 5, p. 2343-2356. \\ (C) 2007. The American Astronomical Society. All rights reserved.
}

The copyright holder has granted permission for posting the article here. 


\title{
DETERMINATION OF THE PHYSICAL CONDITIONS OF THE KNOTS IN THE HELIX NEBULA FROM OPTICAL AND INFRARED OBSERVATIONS ${ }^{1}$
}

\author{
C. R. O’DELL \\ Department of Physics and Astronomy, Vanderbilt University, Nashville, TN 37235, USA; cr.odell@vanderbilt.edu \\ W. J. HENNEY \\ Centro de Radioastronomía y Astrofísica, Universidad Nacional Autónoma de México, 58090 Morelia, Michaoacán, Mexico \\ AND \\ G. J. Ferland \\ Department of Physics and Astronomy, University of Kentucky, Lexington, KY 40506, USA \\ Received 2006 November 13; accepted 2007 January 18
}

\begin{abstract}
We use new Hubble Space Telescope and archived images to clarify the nature of the ubiquitous knots in the Helix Nebula, which are variously estimated to contain a significant to majority fraction of the material ejected by its central star. We employ published far-infrared spectrophotometry and existing $2.12 \mu \mathrm{m}$ images to establish that the population distribution of the lowest rovibrational states of $\mathrm{H}_{2}$ is close to the distribution of a gas in local thermodynamic equilibrium at $988 \pm 119 \mathrm{~K}$. In addition, we present calculations that show that the weakness of the $\mathrm{H}_{2} 0-0 S(7)$ line is not a reason for making the unlikely-to-be-true assumption that $\mathrm{H}_{2}$ emission is caused by shock excitation. We derive a total flux from the nebula in $\mathrm{H}_{2}$ lines and compare this with the power available from the central star for producing this radiation. We establish that neither soft X-rays nor 912-1100 $\AA$ radiation has enough energy to power the $\mathrm{H}_{2}$ radiation - only the stellar extreme-ultraviolet radiation shortward of 912 Å does. New images of the knot 378-801 in the $\mathrm{H}_{2} 2.12 \mu \mathrm{m}$ line reveal that the $2.12 \mu \mathrm{m}$ cusp lies immediately inside the ionized atomic gas zone. This property is shared by material in the "tail" region. The $\mathrm{H}_{2} 2.12 \mu \mathrm{m}$ emission of the cusp confirms previous assumptions, while the tail's property firmly establishes that the tail structure is an ionization-bounded radiation shadow behind the optically thick core of the knot. A unique new image of a transitional region of the nebula's inner disk in the He II $\lambda 4686$ line fails to show any emission from knots that might have been found in the $\mathrm{He}^{++}$core of the nebula. We also reexamined high signal-to-noise ratio ground-based telescope images of this same inner region and found no evidence of structures that could be related to knots.
\end{abstract}

Key words: planetary nebulae: individual (Helix Nebula, NGC 7293)

\section{INTRODUCTION}

The dense knots that populate the closest bright planetary nebula, NGC 7293 (the Helix Nebula), must play an important role in mass loss from highly evolved intermediate-mass stars and therefore in the nature of enrichment of the interstellar medium (ISM) by these stars. It is likely that similar dense condensations are ubiquitous among the planetary nebulae (O'Dell et al. 2002), as the closest five planetary nebulae show similar or related structures. They are an important component of the mass lost by their host stars, for the characteristic mass of individual knots has been reported as $\geq 10^{-5} M_{\odot}$ (from CO emission; Huggins et al. 2002), $5.6 \times 10^{-5} M_{\odot}$ (from the dust optical depth determination by Meaburn et al. [1992], adjusted for the improved distance), and about $3.8 \times 10^{-5} M_{\odot}$ (O’Dell \& Burkert [1997], again from the dust optical depth but with better spatial resolution), and the number of them has been variously estimated to be from 3500 (O'Dell \& Handron 1996) from optical observations to much larger numbers (23,000: Meixner et al. 2005, hereafter MX05; 20,000-40,000: Hora et al. 2006, hereafter H06) from infrared imaging. Therefore, these condensations contain a significant fraction a majority of all the material ejected. It is an extremely

\footnotetext{
${ }^{1}$ Based on observations with the NASA/ESA Hubble Space Telescope, obtained at the Space Telescope Science Institute, which is operated by the Association of Universities for Research in Astronomy, Inc., under NASA contract NAS5-26555.
}

important point to understand whether the ISM is being seeded by these knots and whether they survive long enough to be important in the general properties of the ISM and also the process of formation of new stars. To understand those late phases, long after the knots have escaped the ionizing environment of their central stars, one must understand their characteristics soon after their formation - which is the subject of this study.

There has been a burst of interest in the Helix Nebula and its knots, beginning with the lower resolution ground-based study of Meaburn et al. (1992) and the Hubble Space Telescope (HST) images at better than 0.1" resolution (O’Dell \& Handron 1996; O'Dell \& Burkert 1997) in the optical window. The entire nebula has been imaged in the $\mathrm{H}_{2} v=1-0 \mathrm{~S}(1) 2.12 \mu \mathrm{m}$ line at scales and resolutions of about $4^{\prime \prime}$ (Speck et al. 2002) and 1.7" pixel $^{-1}$ (H06), while Huggins et al. (2002) have studied one small region at $1.2^{\prime \prime}$ resolution, and the NIC3 detector of the NICMOS instrument of the HST has been used by Meixner et al. (2004; MX05) to sample several outer regions at about $0.2^{\prime \prime}$ resolution. A lower resolution $\left(2^{\prime \prime}\right)$ study in the longer wavelength $0-0$ rovibrational lines has imaged the entire nebula with the Spitzer Space Telescope (H06), extending a similar investigation by Cox et al. (1998, hereafter C98) at 6" pixel $^{-1}$ with the Infrared Space Observatory. Radio observations of the CO (Huggins et al. 2002; Young et al. 1999) and $\mathrm{H}_{\mathrm{I}}$ (Rodríguez et al. 2002) emission have even lower spatial resolution, but the high spectral resolution allows one to see emission from individual knots. 
The three-dimensional model for the Helix Nebula has also evolved during this time. We now know that the inner part of the nebula is a thick disk of $500^{\prime \prime}$ diameter seen at an angle of about $23^{\circ}$ from the plane of the sky (O'Dell et al. 2004, hereafter OMM04). This disk has a central core of high-ionization material traced by He II emission (4686 $\AA$ ), and a series of progressively lower ionization zones until its ionization front is reached. The more easily visible lower ionization portions of the inner disk form the inner ring of the nebula. There are polar plumes of material perpendicular to this inner disk extending out to at least $940^{\prime \prime}$ (OMM04) to both the northwest and southeast. There is an apparent irregular outer ring that Meaburn et al. (2005, hereafter M05) argued is a thin layer of material on the surface of the perpendicular plumes, whereas OMM04 and O'Dell (2005) argued that this is due to a larger ring lying almost perpendicular to the inner disk.

The nature of the knots has attracted considerable attention. O'Dell \& Burkert (1997) determined the properties using HST WFPC2 emission-line images in $\mathrm{H} \alpha$, [N II], and [O III], while O'Dell et al. (2000, hereafter OHB00) analyzed HST slitless spectra of the bright knot 378-801 in $\mathrm{H} \alpha$ and [N II], an investigation extended in a study (O'Dell et al. 2005, hereafter OHF05) with better slitless images in the same lines and also the [O I] line at $6300 \AA$. We adopt the position-based designation system described in O'Dell \& Burkert (1997) and the trigonometric parallax distance of $219 \mathrm{pc}$ from Harris et al. (2007). The object $378-801$ is the best studied of the knots and the primary target for the program reported on in this paper. At 219 pc distance from the Sun, the $1.5^{\prime \prime}$ chord of the bright cusp surrounding the neutral, central core of $378-801$ is $4.9 \times 10^{15} \mathrm{~cm}$. O'Dell \& Burkert (1997) estimated that the peak density in the ionized cusp is about $1200 \mathrm{~cm}^{-3}$ and the central density of the core, derived from the optical depth in dust, is $4.8 \times 10^{5} \mathrm{~cm}^{-3}$, a number similar to the $\mathrm{H}_{2}$ density of $\geq 10^{5} \mathrm{~cm}^{-3}$ necessary to produce the thermalized population distribution found for the $J$-states within the $v=0$ levels of the electronic $\left(X^{1} \Sigma_{g}^{+}\right)$ground state by C98. C98 determined that two sample regions of knots were close to a population distribution of about $900 \mathrm{~K}$; a similar result is found by an analysis ( $(4.2)$ of new observations (H06) of different regions of knots.

As was argued in O'Dell \& Handron (1996), the knots are neutral condensations ionized on the side facing the central star. López-Martín et al. (2001) have shown that the early apparent discrepancy between the observed and predicted surface brightness of the bright cusps is resolved once one considers the dynamic nature of the flow from the cusp ionization front, which depresses the recombination emission from the ionized gas. The central cores are molecular, being visible in $\mathrm{CO}$ (Huggins et al. 2002) and producing the multiple velocity components one sees in the low spatial resolution-high velocity resolution $\mathrm{CO}$ studies (Young et al. 1999). The $\mathrm{H}_{2}$ emission is produced in a thin layer of material immediately behind the ionized cusp (Huggins et al. 2002). OHB00 showed that the optical structure within the ionized cusp can only be explained if the material is heated on a timescale that is longer than the time for cool material to flow from the ionization front across the width of the cusp. This slow heating rate means that forbidden lines are seen only farther away from the ionization front (because these require energetic electrons to cause their collisional excitation), whereas recombination lines such as $\mathrm{H} \alpha$ arise preferentially from the cool regions closer to the ionization front. A succession of papers (Burkert \& O'Dell 1998; OHB00; OHF05) attempting to simultaneously account for both the ionization and flow of material has produced a general model in which cool material in the molecular central core flows toward the ionization front, is slowly heated, and upon passing through the ionization front is more rapidly heated and accelerated. At first examination the most recent models look satisfactory. However, the models fail because the theoretically expected zone of $900 \mathrm{~K}$ gas has much too low a column density to account for the observed surface brightness in $\mathrm{H}_{2}$ (OHF05). These $\mathrm{H}_{2}$ observations must be telling us about something overlooked in previous models. As we discuss in $\S 4.4$, this missing ingredient seems to be that the earlier models were for a static structure, whereas the knots are actually in a state of active flow.

This paper reports on work intended to clarify the nature of the knots in the Helix Nebula. New observations with all three of the imaging instruments on the HST were made and are described in $\S 2$, then analyzed in $\S 3$. A new and more refined theoretical model is presented in $\S 4$, while these and other recent observations and models are discussed in $\S 5$.

\section{OBSERVATIONS}

In this investigation we draw on both our own new observations made with the HST and published observations made with a variety of telescopes. These observations range from the optical through the infrared.

\subsection{New Observations}

The new HST observations were made during eight orbits over the period 2006 May 22-24 as program GO-10628 (PI: C. R. O'Dell). The Helix Nebula is sufficiently large that we could simultaneously observe it with the three operating imaging instruments: the WFPC2 (Holtzman et al. 1995), the NICMOS (Thompson et al. 1998), and the ACS (Gonzaga et al. 2005). By careful selection of the pointing and orientation of the spacecraft, we were able to sample three regions that are useful for understanding the knots and their structure. In each case the new observations are either unique or of substantially longer exposure time than previous similar observations. The placement of the fields of view is shown in Figure 1.

\subsubsection{NICMOS Images in $\mathrm{H}_{2}$ of the Knot 378-801}

The NICMOS observations were made with the NIC3 camera $\left(256 \times 256\right.$ pixels, each about $0.2^{\prime \prime}$ pixel $\left.^{-1}\right)$, with eight exposures in both the $\mathrm{F} 212 \mathrm{~N}$ (isolating the $2.12 \mu \mathrm{m} \mathrm{H}_{2}$ line and the underlying continuum) and the $\mathrm{F} 215 \mathrm{~N}$ (isolating primarily the underlying continuum, as there are no strong lines in this region) filters. Each of the 16 exposures was of $1280 \mathrm{~s}$ duration. The pointing was changed in a four-position pattern, with steps of $5^{\prime \prime}$. The onthe-fly standard processing image products were our starting point. These images were combined using IRAF ${ }^{2}$ and tasks from the HST data processing STSDAS package provided by the Space Telescope Science Institute (STScI). The method of calibration used in OHF05 was adopted, in which the nebular and instrumental continuum was subtracted using the signal from the $\mathrm{F} 215 \mathrm{~N}$ filter.

The resultant image is shown in Figure 2 along with aligned WFPC2 optical emission-line images from programs GO-5086 and GO-5311. The knot 378-801 is located in the low central region of each image, and details of its bright cusp and tail are analyzed in $\S \S 3.1$ and 3.2. It is notable that all of the $\mathrm{H}_{2}$ cusps have corresponding $\mathrm{H} \alpha$ and [ $\left.\mathrm{N}_{\mathrm{II}}\right]$ counterparts and that all of the

\footnotetext{
${ }^{2}$ IRAF is distributed by the National Optical Astronomy Observatory, which is operated by the Association of Universities for Research in Astronomy, Inc., under cooperative agreement with the National Science Foundation.
} 


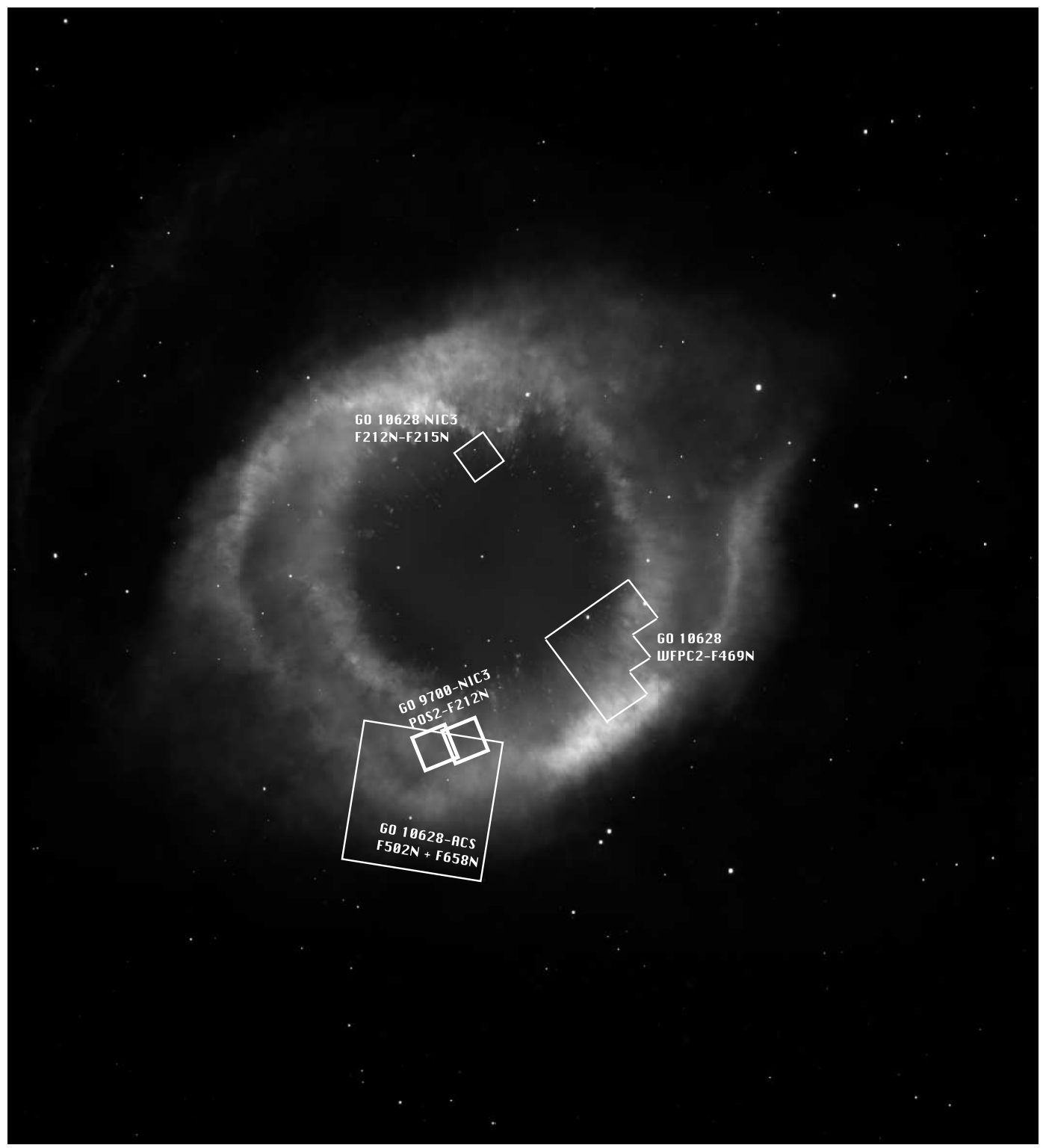

FIG. 1. $-1500^{\prime \prime} \times 1650^{\prime \prime}$ image of the Helix Nebula in $\mathrm{H} \alpha+[\mathrm{N}$ II] from OMM04, showing the fields covered by the three image programs of GO-10628 and also the two NIC3 fields in program GO-9700 that overlap with the GO-10628 ACS field.

$\mathrm{H} \alpha$ and [ $\mathrm{N}$ II] cusps have $\mathrm{H}_{2}$ counterparts on these well-exposed, high-resolution images.

\subsubsection{ACS Images of a South-Southeast Field Previously Observed with NICMOS in $\mathrm{H}_{2}$}

A field to the south-southeast of the central star and falling into the outer-ring portion of the Helix Nebula was imaged with the ACS. Eight exposures of nominally $1200 \mathrm{~s}$ each were made in both the F658N filter (which passes the $\mathrm{H} \alpha$ and [ $\mathrm{N} \mathrm{II]} 26583$ lines equally well) and the F502N filter (dominated by the [O III] 25007 line). The field of view shown in Figure 1 overlaps only slightly with the ACS mosaic built up during the GO-9700 survey with the same filter pairs (OMM04). The signal-to-noise ratio $(\mathrm{S} / \mathrm{N})$ was much higher than in the GO-9700 survey, since that study used total exposures of about $850 \mathrm{~s}$ in both filters.

The images in the four offset pointings were combined using tasks within the STSDAS package. No attempt at absolute calibration was made because of the F658N filter requiring additional observations with the F660N filter, which is dominated by [N II] (O’Dell 2004). The resulting image for F658N is shown in Figure 3. Originally of $0.05^{\prime \prime}$ pixels, it has been averaged into $2 \times 2$ samples in order to increase the $\mathrm{S} / \mathrm{N}$. Since the finest features are larger than the size of the resultant pixels $\left(0.1^{\prime \prime}\right)$, no loss of detail was incurred. The F502N image has a much lower signal and is not presented here, although portions of it are reproduced and discussed in $\S 3.4$.

\subsubsection{WFPC2 Images in the He II Filter of a Southwest Field}

Sixteen exposures of $1100 \mathrm{~s}$ each were made with the WFPC2 F469N filter that isolates the $\mathrm{He}$ II recombination line at $4686 \AA$. The images from the four pointings were combined using the STSDAS package of dither tasks, and the results are shown in Figure 4. For comparison, a matching section of the ACS mosaic that was derived as part of program GO-9700 (OMM04) is also shown.

\subsection{Recently Published Observations}

There are three recent papers that contain observations pertinent to our discussion of the nature of the knots in the Helix 


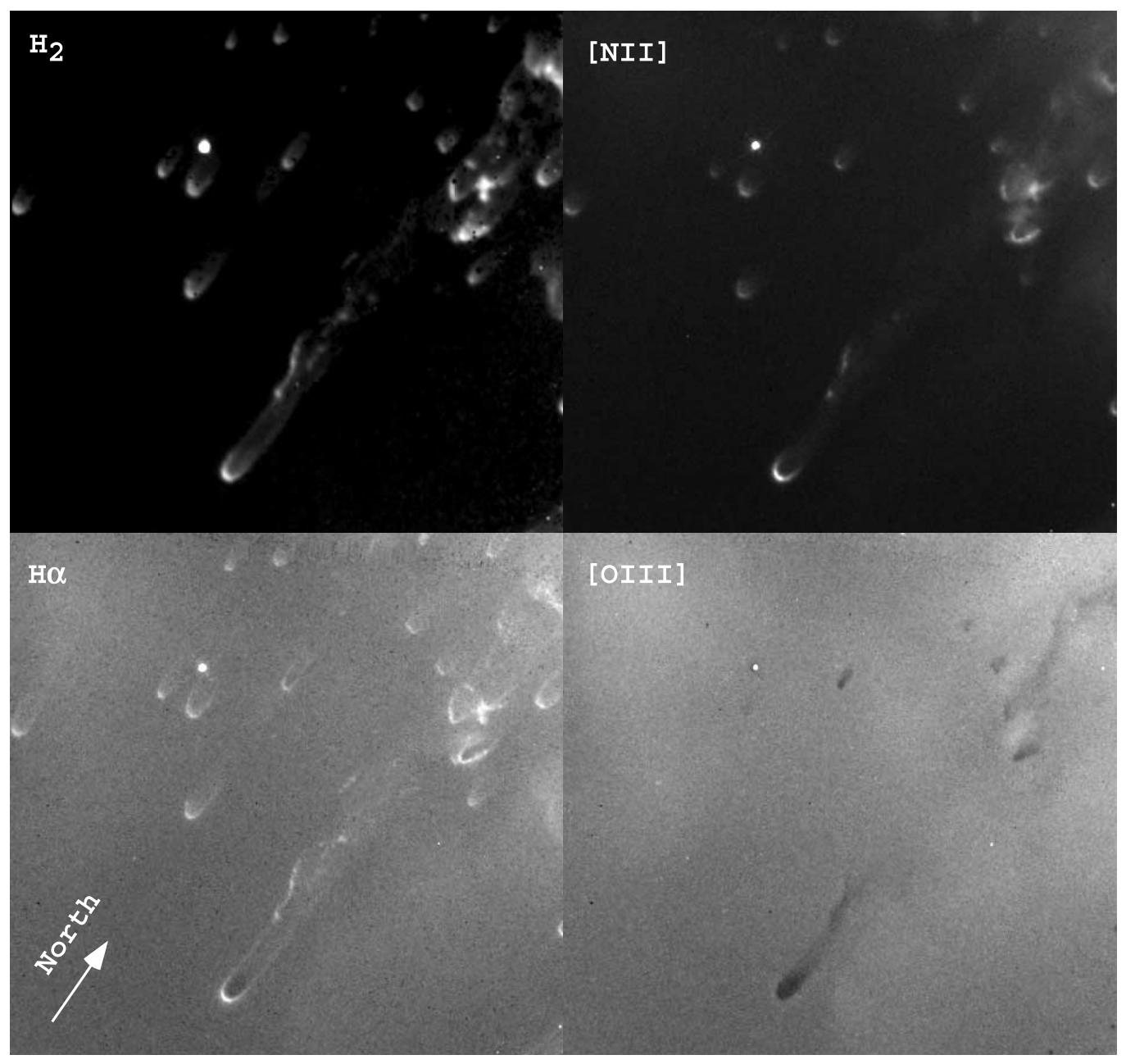

FIG. 2.-Mosaic images depicting the same $47.5^{\prime \prime} \times 44.8^{\prime \prime}$ region targeted with the NICMOS NIC 3 camera. The $\mathrm{H}_{2}$ image is from GO- 10628 , as described in the text, while the optical emission-line images are from WFPC2 programs GO-5086 and GO-5311. The vertical axis is pointed toward a position angle (P.A.) of $35^{\circ}$. The NIC3 images have been scaled to the $0.0996^{\prime \prime}$ pixel $^{-1}$ scale of the WFPC2 camera.

Nebula. These include two space infrared observations and one ground-based study.

\subsubsection{Spitzer Space Telescope Infrared Images and Spectra}

In a recent paper, $\mathrm{H} 06$ presented the results of an extensive observational study of the Helix Nebula. They imaged the object out to the northeast arc (OMM04) with the IRAC camera (Fazio et al. 2004) in four broad filters centered on 3.6, 4.5, 5.8, and $8.0 \mu \mathrm{m}$. These filters are dominated by emission from rovibrational lines within the $v=0$ ground electronic state, although an atomic continuum must be present, in addition to a few collisionally excited forbidden and recombination emission lines. The resolution of these images is about $2^{\prime \prime}$, so one cannot resolve structure within the cusps, but one can see structure along radial lines passing through the bright cusps and their much longer tails. Spectra were obtained with the IRS (Houck et al. 2004) at three positions, two falling in the outer ring at locations north and southwest of the central star and the third location (used for background subtraction) falling directly on the fainter northeast arc feature. They present calibrated fluxes for rovibrational lines of $\mathrm{H}_{2}$ from $0-0 S(7)$ at $5.51 \mu \mathrm{m}$ out to $0-0 S(1)$ at $17.0 \mu \mathrm{m}$. They also present a ground-based image in a filter centered on the $2.12 \mu \mathrm{m} \mathrm{H}_{2}$ line that is of comparable spatial resolution but wider field of view than that of the Speck et al. (2002) study. This image is not flux calibrated but appears to go fainter than the Speck et al. (2002) image.

\subsubsection{HST GO-9700 Images in $\mathrm{H}_{2}$}

In the GO-9700 study that produced a continuous mosaic of ACS images, MX05 also obtained parallel NIC3 images in the F212N filter at six science positions and one sky position. Each position was actually a double exposure of two pointings, which allowed a slight overlap of the NIC3 fields. The total exposure at each position was about $750 \mathrm{~s}$ and double that in the regions of overlap. The method of calibration was different, as only F212N images were obtained (the few F175W images were not useful for calibration of the F $212 \mathrm{~N}$ images). It was assumed that the sky images included all the background signal that needed to be subtracted, which means that the nebular continuum was not subtracted. The NIC3 images are undersampled (the pixel size of $0.2^{\prime \prime}$ is about the same as the telescope's resolution at this wavelength) as in our new observations of the field around 378-801. When comparing the data, one should note that the maximum effective exposure time is about $1500 \mathrm{~s}$ for the GO-9700 F212N images, while in our study it is $10,240 \mathrm{~s}$. As discussed in $\S 3.4$ our ACS field overlaps with much of the MX05 position 2 field, allowing a more meaningful comparison of optical and infrared images of this region than was possible in the MX05 study.

\section{ANALYSIS OF THE OBSERVATIONS}

These new images of the Helix Nebula with three different HST cameras provide new information on a number of subjects related 


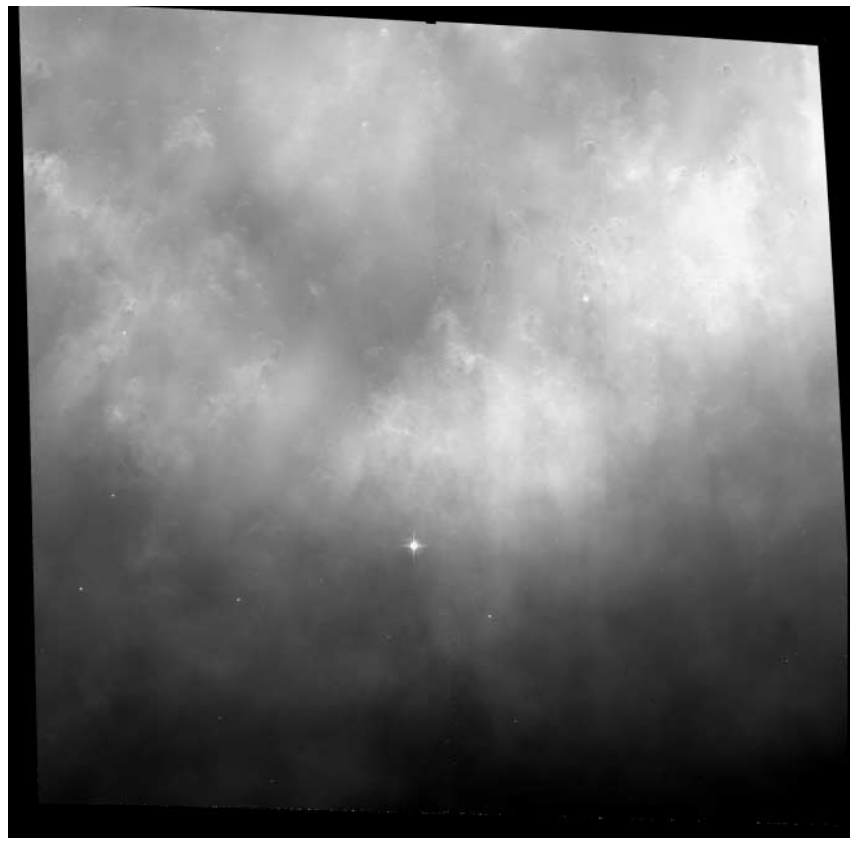

FIG. 3.-High-contrast $215^{\prime \prime} \times 213^{\prime \prime}$ image of the ACS region shown in Fig. 1, with the vertical axis pointed toward P.A. $=350^{\circ}$. It demonstrates that bright cusp knots are found in the outer ring of the nebula, in addition to the inner-ring knots that dominate the field covered by the GO-9700 mosaic.

to the nature and formation of the knots. The NICMOS $\mathrm{H}_{2}$ image allows a discussion of the cusp and tail structure, the He II image places constraints on the location of the knots to the southwest, and the ACS images allow a more complete comparison of early $\mathrm{H}_{2}$ images to the south and southeast with comparable resolution optical emission-line images.

\subsection{Stratification in the Bright Cusp of 378-801}

The new NIC3 $\mathrm{H}_{2} 2.12 \mu \mathrm{m}$ line observations were scaled to the same pixel size as the WFPC2 $\left(0.0996^{\prime \prime}\right.$ pixel $\left.^{-1}\right)$ using bi- linear interpolation and were carefully aligned. Although this region has been imaged numerous times in this line, this is clearly the best image in terms of both its resolution and signal. The three primary optical-line images and the new $\mathrm{H}_{2}$ image are shown in Figure 2. In order to make a quantitative analysis, the images were rotated, and a sample 3 pixels wide along the axis of the cusp-core tail was made. The results are shown in Figure 5, where the peak value of the $\mathrm{H}_{2} 2.12 \mu \mathrm{m}$ and [ $\mathrm{N}$ II] emission is normalized to unity as are the outer portions of the [O III] profiles.

The optical-line results are similar to those found by O'Dell \& Burkert (1997), Burkert \& O’Dell (1998), O’Dell et al. (2000), and OHF05 in that the ionization occurring farthest from the knots' ionization front ([O III]) is weak and extended; this intrinsic low brightness allows one to see the core of the knot in silhouette against the background nebular emission in this line. Although the extinction peaks in the core of the knot, it extends to about $5^{\prime \prime}$ from the bright cusp. The [N II] emission is strong and displaced $\left(0.05^{\prime \prime}\right)$ away from the ionization front with respect to the $\mathrm{H} \alpha$ emission. One sees extinction in $\mathrm{H} \alpha$ from the core out to almost the same distance as in [O III]. The lack of apparent extinction in $[\mathrm{N}$ II] must be due to there being relatively more emission in that line in the sheath of ionized gas surrounding the shadow of the knot. The full width at half maximum (FWHM) of the $\mathrm{H} \alpha$ and the [N $\mathrm{N}_{\mathrm{II}}$ ] images is $0.48^{\prime \prime}$, and since the FWHM of the stars in the field of view is $0.25^{\prime \prime}$, quadratic subtraction of this instrumental component leaves an intrinsic line width in those emission lines of $0.41^{\prime \prime}$. The FWHM of the $\mathrm{H}_{2}$ line is $0.61^{\prime \prime}$ and the nearby star's is $0.42^{\prime \prime}$, leaving an intrinsic FWHM for $\mathrm{H}_{2}$ of $0.44^{\prime \prime}$ with a peak displaced $0.11^{\prime \prime}$ toward the core of the knot from the $\mathrm{H} \alpha$ peak. The FWHM corresponds to a length of $1.40 \times$ $10^{15} \mathrm{~cm}$ and the displacement to $3.5 \times 10^{14} \mathrm{~cm}$. The ionized line characteristics are similar to those found in previous studies, but we have added here the important characteristic of the small but certain displacement of the broader $\mathrm{H}_{2}$. The earlier $\mathrm{H}_{2}$ studies lacked the resolution to determine this characteristic, or in the case of MX05, lacked the high-resolution optical lines necessary for the comparison.
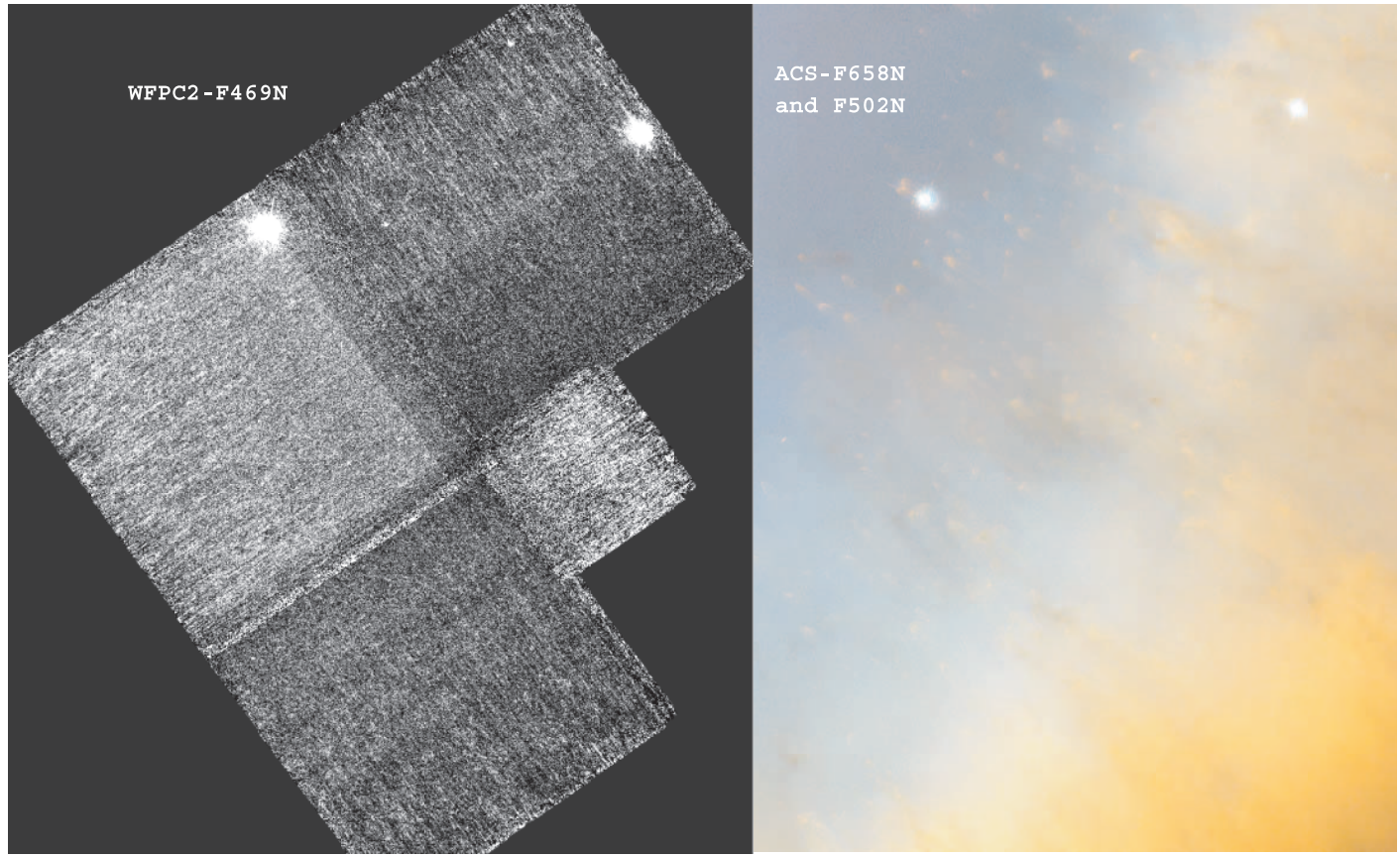

FIG. 4. - Left: $167^{\prime \prime} \times 204^{\prime \prime}$ WFPC2 F469N image with north as the vertical axis, the result of combining 16 dithered images. Right: Same section of the nebula as in the left panel, from a mosaic of ACS images (OMM04) with $\mathrm{H} \alpha+[\mathrm{N} \mathrm{II}]$ as red, an average of $\mathrm{H} \alpha+[\mathrm{N} \mathrm{II}]$ and [O III] as green, and [O III] as blue. No indication of features showing He II $\lambda 4686$ emission is seen. 


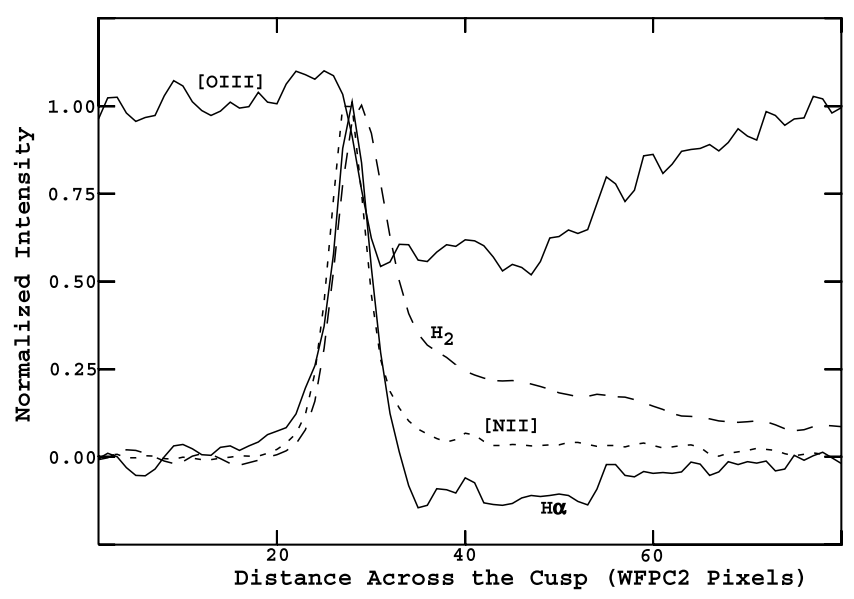

FIG. 5.- Normalized intensity of a sample along the axis of knot 378-801, shown for three optical lines and the $\mathrm{H}_{2} 2.12 \mu \mathrm{m}$ infrared line. The $2.12 \mu \mathrm{m}$ image has lower resolution (about 2 pixels), and a comparison of the appearance in the different lines is discussed in the text.

The calibrated peak surface brightness in the cusp of 378-801 is $1.8 \times 10^{-4}$ ergs cm ${ }^{-2} \mathrm{~s}^{-1} \mathrm{sr}^{-1}$ in the $\mathrm{H}_{2} 2.12 \mu \mathrm{m}$ line. This agrees well with the peak value of $1.0 \times 10^{-4}$ ergs $\mathrm{cm}^{-2} \mathrm{~s}^{-1} \mathrm{sr}^{-1}$ found by Huggins et al. (2002), for which they used the calibration of Speck et al. (2002) and utilized a spatial resolution that would not have recognized the narrowness of the peak.

\subsection{Stratification in the Tail of 378-801}

The well-defined tail in 378-801 is primarily formed by a shadow in the ionizing Lyman continuum ( $\mathrm{LyC}$ ) radiation cast by the optically thick core, with illumination occurring by diffuse (recombination) LyC photons and direct radiation grazing the edge of the core. The first-order theory describing this situation was presented by Canto et al. (1998) and applied to the tails of the Helix Nebula and the shadows behind the Orion Nebula proplyds soon after (O'Dell 2000). OHF05 discussed the structure in the tail of 378-801 within the context of this theory and its next-order refinements (Wood et al. 2004) but were unable to explain the details of what was being seen. $\mathrm{H}_{2}$ in the tails was first detected by Walsh \& Ageorges (2003), and we are able to establish with our new observations where this emission arises.

We present in Figure 6 results from traces across the tail of $378-801$, extending from $3.1^{\prime \prime}$ to $6.0^{\prime \prime}$ behind the peak of the cusp

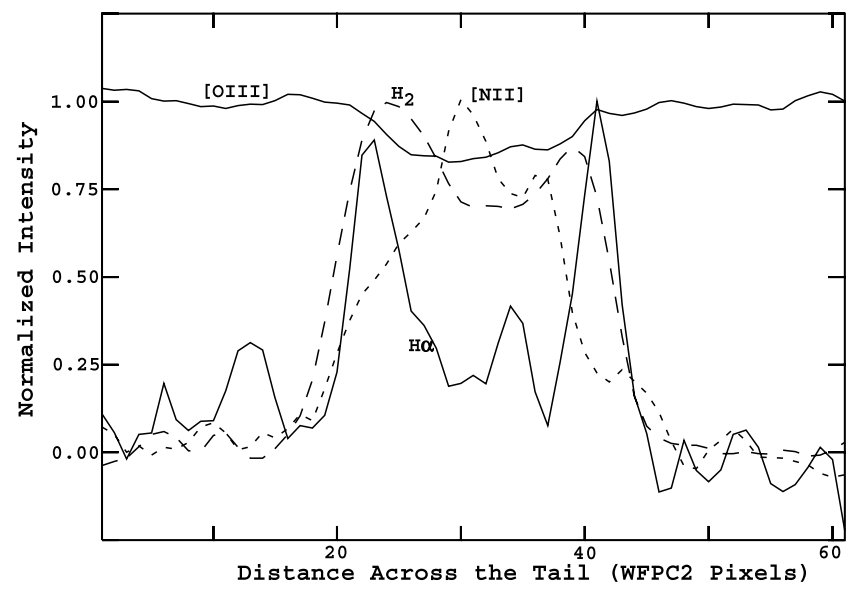

FIG. 6.-Cross section of a tail region of knot 378-801, with the radiation in each line normalized to unity. The details of the sample and interpretation are presented in the text.

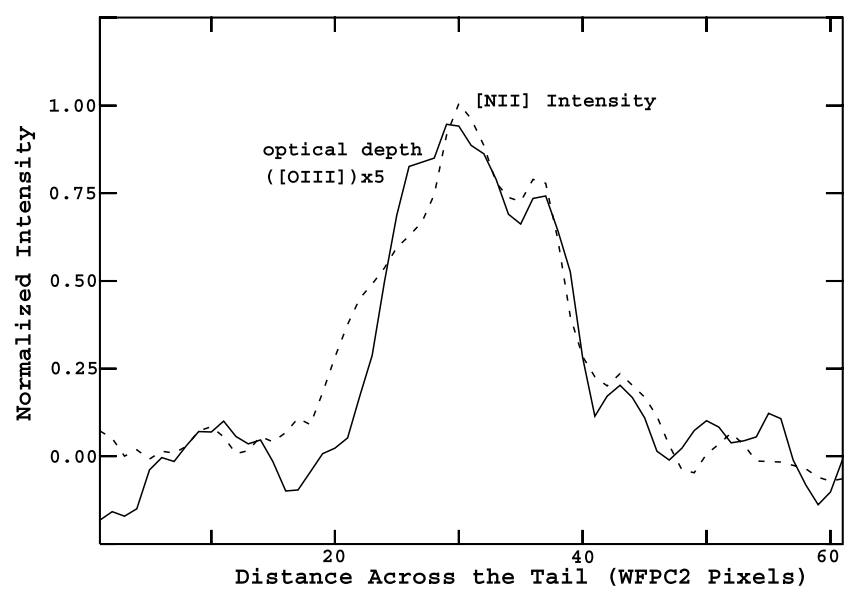

FIG. 7.- Normalized surface brightness of the tail sample of Fig. 6, shown together with the optical depth (multiplied by 5) determined from the [O III] profile. The similar distribution of each argues that the [ $\mathrm{N} \mathrm{II}]$ emission is caused by scattering of nebular [N II] emission, as discussed in the text.

in $\mathrm{H} \alpha$. This region does not extend as far as the partially obscured knot lying on the east side of the tail with its cusp $8.3^{\prime \prime}$ beyond 378-801's $\mathrm{H} \alpha$ cusp. One sees that there is a well-defined signature of a limb-brightened sheath in both $\mathrm{H}_{2} 2.12 \mu \mathrm{m}$ and $\mathrm{H} \alpha$, with the peaks of the $\mathrm{H}_{2}$ emission occurring inside the $\mathrm{H} \alpha$, as expected if the $\mathrm{H} \alpha$ is associated with a local ionization front. Since the gas ionized by diffuse radiation should be much cooler than the directly illuminated nebular gas, the $\mathrm{H} \alpha$ emissivity would be high and the ionized sheath is well defined. These observations establish that conditions in the tail do allow an ionization front to form, while Cantó et al. (1998) and O'Dell (2000) had expected the shadowed region to be fully ionized. It is not surprising that no [O III] emission is seen; rather, the dust in the tail, concentrated in the middle of the tail (as noted by OHF05), causes extinction of the background nebular light. The apparent quandary, noted in OHF05, is that the [N II] emission appears to come from inside the ionized sheath of the tail. We already noted that the $\mathrm{H} \alpha$ and $\mathrm{H}_{2}$ structure indicates that an ionization boundary occurs at the edge of the radiation shadow, so there is an apparent contradiction in finding ionized nitrogen emission originating inside an ionization front.

This contradiction is removed by comparison of the [ $\mathrm{N}$ II] emission and the optical depth, as determined by the [O III] image. Figure 7 shows a comparison of the dust optical depth and the [N II] intensity. The similarity of the distribution of the optical

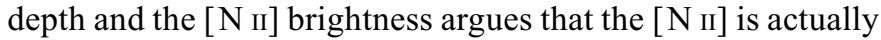
nebular or cusp light scattered by the only marginally optically thick (peak value: $\tau=0.2$ columns of dust). This feature is easy to see because the expected low electron temperature of the sheath's ionization front would suppress the collisionally excited [ $\mathrm{N}$ II]. If our interpretation of [ $\mathrm{N}$ II] is correct, then there should be a similar component of scattered $\mathrm{H} \alpha$ radiation. This may be what somewhat fills-in the region between the two limb-brightened components (in addition to the low level of surface brightness expected when examining a thin shell). In the cusp [N II] is stronger than $\mathrm{H} \alpha$ emission, whereas in this part of the nebula the opposite is true. This point argues that much of the scattered [N II] emission arises from the nearby bright cusp rather than the surrounding nebula.

The distribution along the tail core is discussed in detail in OHF05 (see their $\S 4.1 .2$ ), and only a few comments need to be added here. The question is complex because the knots seem to originate near the nebular ionization front, then are shaped by 


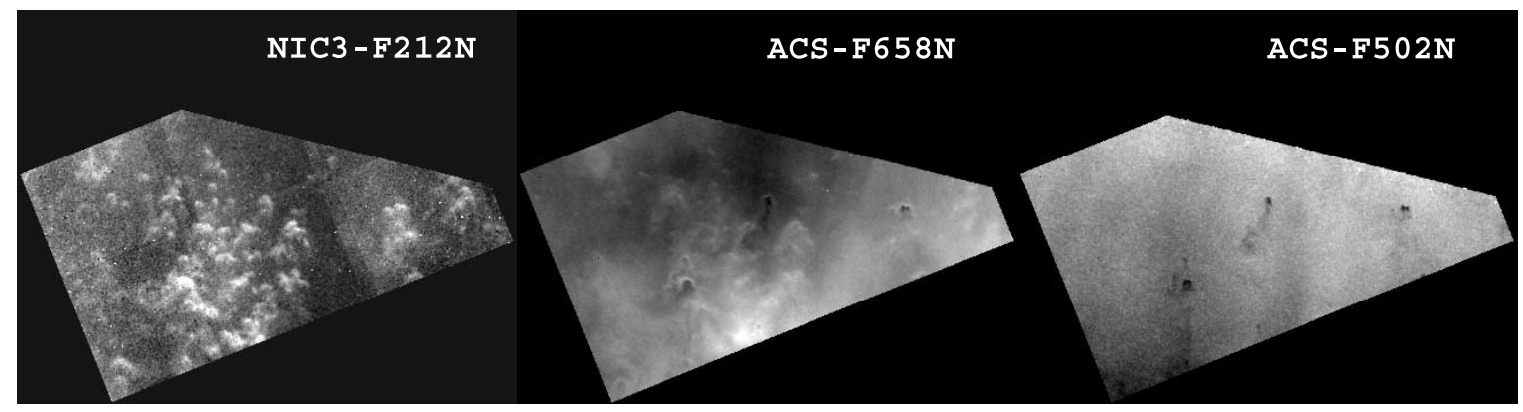

FIG. 8.-88" wide field of overlapping ACS (GO-10628) and NIC3 (GO-9700) images, with north up. The $\mathrm{H}_{2}$ cusps of the knots are much more visible than the combined $\mathrm{H} \alpha+\left[\mathrm{N}\right.$ II] ionized cusps. However, close examination shows an ionized gas feature corresponding to each $\mathrm{H}_{2}$ feature. This is not true for the [O III] F502N features, which require that the knot be located in the foreground. The faint circular features are artifacts of the imaging system.

the radiation field as the ionization front expands beyond them (O'Dell et al. 2002). This means that material in the shadowed region never would have seen direct ionizing radiation and could represent pre-knot material from the planetary nebula's photondominated region (PDR). The other source of tail material could be neutral gas accelerated outward by the rocket effect (e.g., Mellema et al. 1998). Unfortunately, the high velocity resolution study of CO by Huggins et al. (2002) does not really illuminate the question. Their angular resolution was a Gaussian beam of $7.9^{\prime \prime} \times 3.8^{\prime \prime}$, with the long axis aligned almost along the axis of the tail of 378-801. Since there was a strong CO component coming from the partially shadowed knot lying $8.3^{\prime \prime}$ beyond 378 801 's $\mathrm{H} \alpha$ bright cusp, this means that there is not a clear resolution of the $\mathrm{CO}$ emission from the core of 378-801 and the partially shadowed knot. As a result, one cannot hope to interpret the small differences in the position of the peak emission at different velocities as core-tail differences. This could be done with higher spatial resolution $\mathrm{CO}$ observations.

\subsection{An Unsuccessful Search for He II Emission in the Southwest Knots}

Figure 4 shows our deep He II images alongside the same field covered at comparable resolution with the ACS in $\mathrm{H} \alpha+[\mathrm{N}$ II $]$ and [O III]. A detailed comparison of the two images indicates that there is no case of a $\mathrm{He}$ II feature corresponding to a $\mathrm{H} \alpha+[\mathrm{N}$ II] or [O III] feature, nor any He II-only features. The part of the WFPC2 field closest to the central star is $144^{\prime \prime}$ distance. The profile of the He II core of the central disk - to which the knots in this part of the nebula belong (OMM04), derived from a wide-fieldof-view He II image (O'Dell 1998) - shows that the core is down almost to $50 \%$ of its peak emission at this distance. If any knots actually occur within the nebula's He II core, we would expect that in the simplest knot models, we would see a He II cusp outside the [O III] zone of each knot, but this is not the case. However, the basic model is not that simple.

The detailed models of OHB00 and OHF05 show that the normal progression of ionization states in the cusp are preserved; that is, closest to the ionization front there is an $\mathrm{He}^{0}+\mathrm{H}^{+}$zone, outside of which there is a $\mathrm{He}^{+}+\mathrm{H}^{+}$zone, and outside of that a $\mathrm{He}^{++}+\mathrm{H}^{+}$zone. In a nearly constant electron temperature nebula, the innermost zone is best traced by the [N II] emission, the next zone by the [O III] emission, and the outermost zone by the He II emission. Things are not so simple in the case of the knots. As the gas flows through the cusp it is heated, only slowly, so that the collisionally excited [ $\mathrm{N}$ II] emission peaks farther out, where the gas is hotter, more than making up for the lower fraction of $\mathrm{N}^{+}$ions. By the time that the second zone is reached, the density has dropped significantly and the [O III] emission is broad and weak. One would expect to find a He II zone associated with a knot only if the knot lies within the nebula's He II core. This He II zone would be quite weak because the density of the knot's gas would have been greatly decreased this far out. Moreover, the gas has probably nearly reached the temperature of the nebula, and these higher temperatures suppress the emission of this recombination line. This means that it will be hard to actually detect by their He II emission any knots within the He II core of the nebula. Probably the strongest evidence that no knots exist in the He II core lies in the fact that we do not see any objects in extinction in any of the observed emission lines.

\subsection{Comparison of the ACS Images in the South-Southeast with Earlier NICMOS $\mathrm{H}_{2}$ Images}

As noted in $\S 2.1 .2$, our ACS field overlapped with one of the double pointings made with NIC3 in F212N as part of program GO-9700 (MX05). MX05 compared their F212N images with the corresponding five fields in OMM04, in which the resolution was about $1^{\prime \prime}$, and ground-based images were used because the HST ACS mosaic did not extend out this far. This factor-of-5 difference in resolution made it difficult to draw firm conclusions about differences and similarities of appearance. They did, however, conclude that even their short (750 s) overlapping double exposures were sufficient to establish that the knot cusps were more visible in $\mathrm{H}_{2}$. In $\S 2.1 .1$ we showed that in the vicinity of $378-801$ the knots are equally visible in both the ionization cusps and the $\mathrm{H}_{2}$ cusps.

In Figure 8 we see a comparison of the GO-9700 F212N $\left(\mathrm{H}_{2}\right)$ images with our new ACS images. There is an excellent correlation of appearance, although the contrast of the $\mathrm{H}_{2}$ emission above the essentially zero nebular background is higher than in F658N $(\mathrm{H} \alpha+[\mathrm{N} \mathrm{II}])$ and as usual the knots are only easily seen in the F502N ([O $\mathrm{III}]$ ) when the knot is in the foreground and can be seen in extinction against the background nebular emission. After considering the flexibility of display of the high-S/N $\mathrm{H} \alpha+\left[\mathrm{N}_{\mathrm{II}}\right]$ images, it is difficult to support the conclusion that $\mathrm{H}_{2} 2.12 \mu \mathrm{m}$ images are a better way of searching for knot cusps, except for any regions in which there is high obscuration.

\subsection{Variation in the $\mathrm{H}_{2}$ Cusps with Distance from the Central Star}

With the new $\mathrm{H}_{2} 2.12 \mu \mathrm{m}$ NIC3 images in the current program (GO-10628) and the earlier MX05 study with shorter exposures, there is a larger and -we hope-representative sample of resolved $\mathrm{H}_{2}$ cusps over a wide range of stellar distances $(\phi)$. To look for systematic differences, we have identified isolated cusps in each of the available fields. We selected the three closest knots in the GO-10628 field, three in MX05's positions 1 and 2, and 
two in MX05's positions 3 and 4, with no isolated cusps being available in MX05's position 5. In each case we measured the surface brightness at the peak of the $\mathrm{H}_{2}$ cusp, the approximate chord across the knot's center, and the width of the $\mathrm{H}_{2}$ cusp, and determined $\phi$. The GO-10628 and MX05 positions $1-4$ had average $\phi$-values of $139^{\prime \prime}, 290^{\prime \prime}, 278^{\prime \prime}, 375^{\prime \prime}$, and $464^{\prime \prime}$, respectively. The average surface brightnesses (in ergs $\mathrm{cm}^{-2} \mathrm{~s}^{-1} \mathrm{sr}^{-1}$ ) of the cusp peaks were $1.37 \times 10^{-4}, 7.5 \times 10^{-5}, 8.5 \times 10^{-5}, 4.9 \times$ $10^{-5}$, and $4.5 \times 10^{-5}$. The average cusp widths were $0.6^{\prime \prime}, 0.3^{\prime \prime}$, $0.5^{\prime \prime}, 0.8^{\prime \prime}$, and $1.1^{\prime \prime}$. The average chord values were 1.5", $2.5^{\prime \prime}$, $2.2^{\prime \prime}, 2.4^{\prime \prime}$, and $2.7^{\prime \prime}$.

The most pronounced change in a knot characteristic occurs in the cusp peak surface brightness, dropping roughly linearly with $\phi$, with the cusp width growing slowly and the chord width more steadily with $\phi$. The physical interpretation of these patterns awaits a better understanding of individual knots. It should be pointed out that the relative physical distances from the stars are likely to be increasing more rapidly than the relative values of $\phi$. This is because in the three-dimensional model of the Helix Nebula in OMM04, the main disk is inclined at an angle about $23^{\circ}$ out of the plane of the sky, with the northwest side closer to the observer, while the outer disk is inclined about $53^{\circ}$ out of the plane of the sky, with the south-southeast side closer to the observer. Objects associated with the inner disk would have a distance multiplication factor of 1.09 and those in the outer disk a multiplication factor of 1.66 . The objects in the GO-10628 NIC3 field are almost certainly associated with the inner disk. MX05's positions 1 and 2 could belong with either system (accurate radial velocities would determine this), and their positions 3 and 4 are almost certainly associated with the outer disk.

\subsection{Comparison of the Structure of the Knots} in $2.12 \mu \mathrm{m}$ and in $\mathrm{H} \alpha$

Figure 2 demonstrates the remarkable similarity in appearance of the knots in $\mathrm{H} \alpha$ and in our F212N $(2.12 \mu \mathrm{m})$ images, and we discuss the quantitative properties of $378-801$ in $\S \S 3.1$ and 3.2. We have investigated the similarities of the knots by selecting the nine objects (including 378-801) within the NIC3 field of view that are sufficiently isolated to allow a good background subtraction. The peak surface brightness in each cusp was derived in both $2.12 \mu \mathrm{m}$ and $\mathrm{H} \alpha$ for a sample that was 3 WFPC2 pixels wide $\left(0.3^{\prime \prime}\right)$. The peak surface brightness in the tail of each object was determined in a sample that was 11 WFPC2 pixels wide $\left(1.1^{\prime \prime}\right)$ across the tail, the closer end of the sample being 20 WFPC2 pixels $\left(2.0^{\prime \prime}\right)$ displaced from the tip of the bright cusp. We used the $2.12 \mu \mathrm{m}$ calibration described in $\S 2.1 .1$ and the $\mathrm{H} \alpha$ calibration of O'Dell \& Doi (1999), expressing the surface brightnesses in units of photons $\mathrm{cm}^{-2} \mathrm{~s}^{-1} \mathrm{sr}^{-1}$. The average cusp surface brightness ratio of $2.12 \mu \mathrm{m}$ to $\mathrm{H} \alpha$ was $5.5 \pm 1.0$. The average surface brightness ratio for the tail compared with the cusp was $0.23 \pm 0.08$ in $2.12 \mu \mathrm{m}$ and $0.17 \pm 0.05 \mathrm{in} \mathrm{H} \alpha$. This means that in the cusp the $\mathrm{H}_{2} 2.12 \mu \mathrm{m}$ line alone is putting out more than 5 times as many photons as in $\mathrm{H} \alpha$ and that the contrast between the tail and cusp may be slightly higher (the ratio is lower) in $\mathrm{H} \alpha$ than in $2.12 \mu \mathrm{m}$. The remarkable similarity of the knots in a recombination line that follows photoionization of atomic hydrogen and emission in a molecular line heretofore assumed to be the result of radiative pumping between electronic states is discussed in $\S 4.5$.

\section{DISCUSSION}

Our understanding of the physics of the knots has evolved with a better understanding of a model that satisfactorily explains the knots. The initial discrepancy between the cusp surface bright- ness and the simplest photoionization model (O'Dell \& Handron 1996) was resolved by López-Martín et al. (2001) when it was shown that the advection-dominated nature of the flow through the knot ionization fronts leads to a total rate of recombination in the ionized gas that is significantly below what is predicted from naive models of ionization equilibrium. The peculiar photoionization structure of $\mathrm{H} \alpha$ and [N $\mathrm{NI}$ ] emission can also be understood in similar terms - the heating timescale of the ionized gas is comparable to the dynamic timescales for flow away from the knot surface, leading to resolvable temperature gradients that strongly affect the relative distribution of recombination-line and collisional-line emission. The most refined model is that of OHF05, which included both the effects of the radiation field and the hydrodynamic expansion of the knot's ionization front. It is probably accurate to say that the photoionized portions of the knots are now adequately understood, or at least that the models are broadly consistent with the best observations.

The structure in the tails is only beginning to be understood. To the first order, the tails are the effects of radiation shadows in the dominant ionizing species, the LyC photons (Cantó et al. 1998; O’Dell 2000). With this paper ( $\$ 3.2$ ) we have now determined that the well-observed tails are ionization bounded, with $\mathrm{H}_{2}$ sheaths inside the zone of ionized gas that occurs at the edge of the LyC shadow. The inner part of the tail is dense enough in dust to scatter surrounding nebular light, although suggesting that the origin of this material arises from the original process that forms the knots or that the material moves back from the knot remains uncertain.

The greatest quandary surrounds the explanation of the $\mathrm{H}_{2}$ zone that is observed immediately inside the ionized cusps of the knots. The approximate location of this zone of observed $\mathrm{H}_{2}$ is qualitatively where one would expect it. For reasons given below, it is almost certainly not excited by shocks. Some models (e.g., Natta \& Hollenbach 1998) argue that the heating is by absorption of soft X-rays, while others argue that the excitation mechanism is probably fluorescence, during which non-ionizing photons from the stellar continuum excite molecules to the $B^{1} \Sigma_{u}^{+}$ and $C^{1} \Pi_{u}$ electronic states, which then decay, producing the populations of the ground electronic state that give rise to the observed infrared lines. Within the core of the knot the density is sufficiently high and the temperature sufficiently low that multiple heavier molecules are formed and the observed $\mathrm{CO}$ is simply the most easily observed abundant tracer of these heavy molecules.

An alternative method of exciting the $\mathrm{H}_{2}$ molecules is by shocks. At first this idea seems attractive because planetary nebulae as a class are known to possess high-velocity stellar winds and large-scale mass flows with sufficient energy to excite the lowlying energy states of $\mathrm{H}_{2}$ that give rise to the observed infrared lines. C98 first pointed out that the lack of a stellar wind (CerrutiSola \& Perinotto 1985) rules out excitation by wind-driven shocks. A more complete assessment of shocks as the exciting source was given by OHF05 (see their $\S$ 4.3.2), who show that although $\mathrm{H}_{2}$ is heated sufficiently immediately behind a transient shock, this shock would quickly move through the knot and up the tail. The well-defined location of the $\mathrm{H}_{2}$ emission zones immediately behind the ionized cusps and the ionized sheath of the tail strongly argues that we are dealing with a quasi-stationary process, rather than something quite dynamic, such as shocks.

$\mathrm{H} 06$ based their interpretation of $\mathrm{H}_{2}$ emission on the assumption of shock excitation. Their assumption is based on the weakness of the $\mathrm{H}_{2} 1-1 S(7)$ line, suggesting that the radiative models they use predict strong emission in that line, which they do not observe in their spectra. The shortcomings of that criterion for 
determining that the excitation comes from shocks, rather than radiative processes, is discussed in $\S 4$.1. As we show in $\S 4.2$ the H06 spectra also argue for a high excitation temperature, as found by $\mathrm{C} 98$. The $\mathrm{H} 06$ shock interpretation of the relative population distribution of the $\mathrm{H}_{2}$ energy states used six free parameters, as it required three different shock velocities, each with a different relative intensity. This means that one cannot use the population distribution to confirm that method of excitation.

A key element of understanding the $\mathrm{H}_{2}$ emission is the excitation temperature of the gas. C98 used spectra of the $\mathrm{H}_{2} \mathrm{O}-0$ $S(2)$ to $S(7)$ lines to derive the population of their upper states and found that their two sampled regions matched an excitation temperature of $900 \pm 50 \mathrm{~K}$. We show below $(\S 4.2)$ that the new spectra of $\mathrm{H} 06$ of the $\mathrm{H}_{2} 0-0 S(1)$ to $S(7)$ lines in two additional regions agree with the results of $\mathrm{C} 98$ and support the idea that the $\mathrm{H}_{2}$ emission comes from gas that is much hotter than the $50 \mathrm{~K}$ conditions expected (OHF05) in the core of the knots.

OHF05 demonstrated that even their most detailed PDR models could not explain the high surface brightness in $\mathrm{H}_{2}$ of the knot cusps, an argument first made by C98 from more general considerations. The argument reduces to the fact that the observed surface brightness in $\mathrm{H}_{2} 2.12 \mu \mathrm{m}$ radiation is too high to be explained by the column density of $900 \mathrm{~K} \mathrm{H}_{2}$ that is predicted. OHF05 did not have high-resolution $\mathrm{H}_{2} 2.12 \mu \mathrm{m}$ images of their sample knot (378-801), and a comparison using the results of the new observations reported here is given in $\S 4.5$.

Several papers, including the recent MX05 study, have reported that the surface brightnesses are compatible with the earlier theoretical models of Natta \& Hollenbach (1998), in spite of the fact that those authors point out that the knots do not adhere to their general model and would have a higher surface brightness. A more complete critique of earlier claims of agreement of theory and observations is given in OHF05 (see their $\S$ 4.3.3).

In this section we present the total flux from the central star and nebula in $\S 4.1$, establish that the knots commonly have high excitation temperatures ( $\S 4.2$ ), show that the absence of strong 1-1 $S(7)$ emission is not a strong argument for shock excitation of the $\mathrm{H}_{2}(\S 4.3)$, compare the recent data on $\mathrm{H}_{2}$ emission with the best models ( $\S 4.4)$, determine that there is no evidence for radial features extending into the middle of the nebula ( $(4.5)$, and critique a recent paper that argues for the tails being formed primarily by hydrodynamic processes in $\S$ 4.6.

\subsection{The Total Flux from the Helix Nebula and Its Central Star in Various Energies}

The emission from the nebula is in at least quasi-equilibrium with radiation from the central star. This means that the relative fluxes in various nebular and cusp emission lines and in the stellar continuum impose important constraints that must be observed by the correct model for the cusp $\mathrm{H}_{2}$ emission.

\subsubsection{The Central Star}

The stellar continuum has been well defined down to $1200 \AA$ by Bohlin et al. (1982), who concluded that the star has a luminosity of $120 L_{\odot}$ (corrected to the trigonometric parallax distance) and an effective temperature of $123,000 \mathrm{~K}$. In the long-wavelength end of the continuum, the flux per wavelength interval is very close to $\lambda^{-4}$, as expected when one looks at energies much lower than where the peak emission occurs. This total luminosity corresponds to a flux at Earth of $8.8 \times 10^{-8} \mathrm{ergs} \mathrm{cm}^{-2} \mathrm{~s}^{-1}$.

Natta \& Hollenbach (1998) argued that the $\mathrm{H}_{2}$ is heated by $\mathrm{X}$-rays of greater than $100 \mathrm{eV}$ because only these high-energy photons would penetrate the ionization boundary. There are
TABLE 1

Nominal fluxes at Earth from the Helix Central Star

\begin{tabular}{|c|c|c|c|c|}
\hline \multirow[b]{2}{*}{ WAVELENGTH } & \multirow{2}{*}{$\begin{array}{c}\text { RANGE } \\
(\AA)\end{array}$} & \multicolumn{3}{|c|}{ FLuX (ergs $\left.\mathrm{cm}^{-2} \mathrm{~s}^{-1}\right)$} \\
\hline & & Model $^{\mathrm{a}}$ & Blackbody & Observed \\
\hline X-ray ......................... & $<124$ & $4.2 \times 10^{-11}$ & $1.1 \times 10^{-9}$ & $4 \times 10^{-11}$ \\
\hline EUV …..................... & $124-912$ & $8.3 \times 10^{-8}$ & $8.1 \times 10^{-8}$ & $\ldots$ \\
\hline FUV ....................... & $912-1100$ & $1.9 \times 10^{-9}$ & $2.3 \times 10^{-9}$ & $6.9 \times 10^{-9}$ \\
\hline
\end{tabular}

NotE.-Assuming no intervening absorption, and $L=120 L_{\odot}, T_{\text {eff }}=1.23 \times$ $10^{5} \mathrm{~K}$, and $D=213 \mathrm{pc}$.

${ }^{a}$ Model fluxes are from the $\log g=7$, solar abundance model of Rauch (2003).

two emitters in the high-energy end of the spectrum: the central star and a high-temperature component of about $10^{7} \mathrm{~K}$ (Leahy et al. 1994, 1996; Guerrero et al. 2001). The central-star emission in the $0.1-2.0 \mathrm{keV}$ range is $4 \times 10^{-11} \mathrm{ergs} \mathrm{cm}^{-2} \mathrm{~s}^{-1}$ and the emission from the $10^{7} \mathrm{~K}$ component is $9 \times 10^{-14}$ (Leahy et al. 1994).

The wavelength range for the fluorescent pumping mechanism is from about 912 to $1100 \AA$, as determined by the minimum energy for exciting the Lyman bands and the cutoff imposed by the LyC absorption of hydrogen. Extrapolating the continuum from the slightly longer wavelengths that have been observed gives a total flux in this interval of $6.9 \times$ $10^{-9}$ ergs $\mathrm{cm}^{-2} \mathrm{~s}^{-1}$. If the heating is due to photons above the ionization threshold for neutral hydrogen, then the calculated flux for a $123,000 \mathrm{~K}$ blackbody of $120 L_{\odot}$ in the interval from $13.6 \mathrm{eV}$ through $100 \mathrm{eV}$ is $8.1 \times 10^{-8} \mathrm{ergs} \mathrm{cm}^{-2} \mathrm{~s}^{-1}$, representing the largest amount of power coming from the central star. The observed and predicted properties of the star's flux are summarized in Table 1.

\subsubsection{The Nebula's Emission-Line Flux}

The flux from the entire nebula was determined by O'Dell (1998) to be $F(\mathrm{H} \beta)=3.37 \times 10^{-10} \mathrm{ergs} \mathrm{cm}^{-2} \mathrm{~s}^{-1}$ and $F([\mathrm{O}$ III $] 5007 \AA)=1.94 \times 10^{-9}$ ergs $\mathrm{cm}^{-2} \mathrm{~s}^{-1}$ from narrowband filter images. The proximity of the nebula and its line ratios indicates that the interstellar extinction is low and that little correction is necessary.

We have determined the total flux in the $2.12 \mu \mathrm{m}$ line using the calibrated $2.12 \mu \mathrm{m}$ image of Speck et al. (2002). Stars were edited out by hand, with the local values substituted, and the background was assumed to be found in the west of their field, a region of much lower nebular surface brightness in optical lines (OMM04). This process gave $F(2.12 \mu \mathrm{m})=6.2 \times$

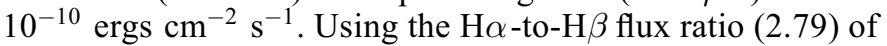
O'Dell (1998) indicates that the number ratio of $2.12 \mu \mathrm{m}$ to $\mathrm{H} \alpha$ for the nebula as a whole is 2.13 , which is less than half the ratio of 5.5 for the cusps in the NIC3 field of view. This is consistent with the fact that almost all of the $\mathrm{H}_{2}$ emission arises from the cusps rather than from the nebula.

C98 have estimated the total flux in the $\mathrm{H}_{2}$ lines falling into their LW2 image to be $2.5 \times 10^{-9}$ ergs $\mathrm{cm}^{-2} \mathrm{~s}^{-1}$. This image contains the lines in the $0-0 S(4)$ through $S(7)$ series. The spectra of C98 and H06 indicate that the strongest line, which is $0-0$ $S(5)$, is $52 \%$ of the total flux of these lines, so the $0-0 S(5)$ line has a total flux from the nebula of $1.3 \times 10^{-9} \mathrm{ergs} \mathrm{cm}^{-2} \mathrm{~s}^{-1}$. If one assumes the line ratios of the H06 study, then the total emission from the nebula in the $0-0 S(1)-S(7)$ lines of $\mathrm{H}_{2}$ becomes $3.8 \times 10^{-9} \mathrm{ergs} \mathrm{cm}^{-2} \mathrm{~s}^{-1}$. If one includes the $2.12 \mu \mathrm{m}$ emission line, this means that the total observed $\mathrm{H}_{2}$ flux is $4.4 \times 10^{-9}$ ergs $\mathrm{cm}^{-2} \mathrm{~s}^{-1}$ and the total $\mathrm{H}_{2}$ emission must be 
more, because there are numerous transitions that have not been observed.

A comparison of the optical recombination plus collisionally excited lines with the $\mathrm{H}_{2}$ emission indicates that a comparable amount of radiation from the nebula is coming out as $\mathrm{H}_{2}$ emission. Since no perceptible $\mathrm{H}_{2}$ emission comes from the nebula, this means that the process producing the $\mathrm{H}_{2}$ emission in the knots is working very efficiently.

Any mechanism seeking to explain the $\mathrm{H}_{2}$ emission must account for $\geq 4.4 \times 10^{-9}$ ergs cm$~^{-2} \mathrm{~s}^{-1}$. This value is much larger than the soft X-ray stellar flux of $4 \times 10^{-11} \mathrm{ergs} \mathrm{cm}^{-2} \mathrm{~s}^{-1}$. The $\mathrm{H}_{2}$ flux is comparable to the 912-1100 $\AA$ total stellar flux of $6.9 \times 10^{-9}$ ergs cm $\mathrm{cm}^{-2} \mathrm{~s}^{-1}$. However, since the fluorescence mechanism operates by absorption of relatively narrow samples within this wavelength range, it appears that this mechanism is also unable to provide enough energy to power the $\mathrm{H}_{2}$ emission. There is certainly enough power available in the stellar continuum if the mechanism depends on absorption of a broad wavelength range rather than on narrow emission lines. This means that $\geq 64 \%$ of the photons with energies less than $13.6 \mathrm{eV}$ would need to be absorbed, or about $\geq 5 \%$ of the $13.6-100 \mathrm{eV}$ radiation. These fractions would have to be larger if the knots are concentrated in a small fraction of the view of the central star. This is certainly the case, as the knots are found exclusively in the lower ionization portions of the main disk of the nebula and in the inner boundary of the outer disk (OMM04). This consideration finally rules out any possibility of the non-ionizing photons being a source of the power.

\subsection{The Population Distribution of the Levels Producing the Observed $\mathrm{H}_{2}$ Lines}

The nature of the population distribution in the upper states that produce the observed infrared $\mathrm{H}_{2}$ emission lines can be a powerful diagnostic in understanding the physical procedures operating in the knots. Therefore, we have determined this distribution using the two available sets of data.

C98 demonstrated that the spectrum in both of their samples closely matched a single excitation temperature of $900 \pm 100 \mathrm{~K}$, using this result to argue that the $\mathrm{H}_{2}$ regions emitting the $0-0 S(2)$ to $0-0 S(7)$ lines had total densities $\geq 10^{5} \mathrm{~cm}^{-3}$. The C98 samples are in the region called the outer ring by OMM04. H06 observed over a slightly larger wavelength range, reporting the detection of the $0-0 S(1)$ line at one of their two positions, but did not present a derived population distribution, probably because they assumed that the population was determined by shocks, as discussed in $\S 4.3$. H06 did not identify where the samples were obtained, except that they were in the "main ring of the nebula." An examination of the Spitzer Space Telescope database shows that both $\mathrm{H} 06$ samples also fall in the region of the outer ring at distances from the central star that are very similar to those of C98.

We have determined the population distribution for each of the four positions for which these studies provide data. H06 presented tabulated line fluxes, including the same lines as the C98 study, with the exception of reporting a flux for the $0-0 S(1)$ line at one of their positions. A plot of their spectra does not include this wavelength region. No errors are reported for their fluxes, and it is impossible to judge the accuracy of the single 0-0 S(1) flux without a spectrum, so we do not use that line in our analysis. C98 did not give tabulated values of their fluxes; however, they too presented plots of the spectra for both regions. These plots were used to measure the line fluxes relative to the strongest line, $0-0 S(5)$. Because of the importance ascribed by H06 to the presence or absence of the 1-1 S(7) line, we carefully looked at its expected wavelength and made a very marginal detection in C98's more southern position. Because the uncertainty of that flux measurement is so great, we do not use it in this analysis of the population distribution.

We have added one additional point in this study by including the $2.12 \mu \mathrm{m}$ line, which is the $1-0 S(1)$ line. Since there is not a matching sample of the nebula with the $2.12 \mu \mathrm{m}$ and $0-0 S(5)$ lines, we have compared the flux in these lines from the entire nebula, using the total fluxes derived in $\S$ 4.1.2. Because of the very different origin of the $1-0 S(1)$ to $0-0 S(5)$ flux ratio, we have not used it in this population distribution analysis, but it is of interest that it appears that the $2.12 \mu \mathrm{m}$ emission is coming from a region with very similar temperature to the other $\mathrm{H}_{2}$ emission.

The ratio of the intensity of an $\mathrm{H}_{2}$ line produced by a transition from an upper state $u$ and lower state $l$ is given by

$$
I_{u l}=N\left(v_{u}, J_{u}\right) A_{u l} h \nu_{u l} .
$$

Any intensity can be converted into a column density from this equation. Most often we deal with a ratio of intensities and thus represent the populations as an excitation temperature $T_{u, 1 ; u, 2}$, which is defined as the temperature that produced the derived population ratio or, implicitly, as

$$
\begin{aligned}
\frac{I_{u l, 1}}{I_{u l, 2}}= & \frac{N_{u, 1} A_{u l, 1} h \nu_{u l, 1}}{N_{u, 2} A_{u l, 2} h \nu_{u l, 2}}=\frac{g_{u, 1} A_{u l, 1} h \nu_{u l, 1}}{g_{u, 2} A_{u l, 2} h \nu_{u l, 2}} \\
& \times \exp \left[\frac{-\chi\left(v_{u, 1}, J_{u, 1}\right)+\chi\left(v_{u, 2}, J_{u, 2}\right)}{T_{u, 1 ; u, 2}}\right],
\end{aligned}
$$

where $\chi$ is the excitation energy (in kelvins). Our sources of molecular data for $\mathrm{H}_{2}$ are given in Shaw et al. (2005). We use excitation energies given by Dabrowski (1984, corrected by E. Roueff [2004], private communication). Transition probabilities are taken from Wolniewicz et al. (1998). Table 2 gives intensities relative to the $0-0 S(5)$ line, which has an upper level of $(v=0, J=7)$. Figure 9 shows the derived column densities relative to the column density of the ( $v=0, J=7)$ level, as a function of the excitation energy $\chi$. The lower half of the figure shows the ratio of column density expressed as an excitation temperature, again using the previous equation. We do not consider in the solutions for the excitation temperature the two lines that are each only marginally detected at a single position or the $2.12 \mu \mathrm{m}$ point derived for the entire nebula. The mean excitation temperatures and standard deviations are also presented in footnotes to Table 2, including the the mean and standard deviation over all the positions.

\subsection{Does the Weakness of the $\mathrm{H}_{2}$ 1-1 S(7) Transition Indicate that $\mathrm{H}_{2}$ Emission Is Powered by Shocks?}

H06 argued that the weakness of the 1-1 S(7) line shows that the $\mathrm{H}_{2}$ emission must be shock-excited rather than photo-excited. Since their subsequent interpretation of the observed features of the nebula are based on this assumption, it merits critical examination. H06 stated that, without presenting detailed proof, the $1-1 S(7)$ line is "normally strong" under photoexcitation. They did not detect this line, but they did detect the nearby $0-0 S(7)$ line. Therefore, we base our discussion on the flux ratio of $1-1 S(7)$ to the $0-0 S(7)$ line. Examination of their spectra (see Fig. 9 of H06) indicates that the flux ratio must be less than about 0.1 . The $1-1 S(7)$ appears to be present in the C98 spectra at a level giving a line ratio of about 0.1 . 
TABLE 2

Relative Line Fluxes and Derived Temperatures for the $\mathrm{H}_{2}$

\begin{tabular}{|c|c|c|c|c|c|c|}
\hline \multirow[b]{2}{*}{ Transition } & \multirow{2}{*}{$\begin{array}{c}\chi \\
(\mathrm{K})\end{array}$} & \multicolumn{5}{|c|}{ Relative Flux in Different Samples ${ }^{a}$} \\
\hline & & Cox $($ W-NW 1-1 S) & $\operatorname{Cox}(\mathrm{NW})^{\mathrm{c}}$ & Hora $(N)^{d}$ & Hora $(\mathrm{SW})^{\mathrm{e}}$ & Full (Nebula) \\
\hline $0-0 S(1)$ & 1015 & $\ldots$ & $\ldots$ & $0.229:$ & $\ldots$ & $\ldots$ \\
\hline $0-0 S(2) \ldots \ldots \ldots \ldots$ & 1681 & 0.088 & 0.091 & 0.132 & 0.11 & $\ldots$ \\
\hline $0-0 S(3) \ldots \ldots \ldots \ldots$ & 2503 & 0.916 & 0.757 & 0.716 & 0.645 & $\ldots$ \\
\hline $0-0 S(4) \ldots \ldots$ & 3474 & 0.319 & 0.368 & 0.296 & 0.277 & $\ldots$ \\
\hline $0-0 S(5)$ & 4586 & 1 & 1 & 1 & 1 & $\ldots$ \\
\hline $0-0 S(6)$ & 5829 & 0.174 & 0.176 & 0.235 & 0.26 & $\ldots$ \\
\hline $1-0 S(1)$ & 6951 & $\ldots$ & $\ldots$ & $\ldots$ & $\ldots$ & 0.47 \\
\hline $0-0 S(7)$ & 7196 & 0.294 & 0.212 & 0.563 & 0.49 & $\ldots$ \\
\hline 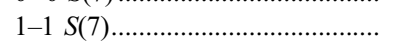 & 12,817 & $0.033:$ & $\ldots$ & $\ldots$ & $\ldots$ & $\ldots$ \\
\hline
\end{tabular}

a The derived $T_{\text {excit }}$ over all samples is $988 \pm 119$.

b The derived $T_{\text {excit }}$ is $935 \pm 110$.

${ }^{c}$ The derived $T_{\text {excit }}$ is $905 \pm 98$.

d The derived $T_{\text {excit }}$ is $1040 \pm 109$.

e The derived $T_{\text {excit }}$ is $1080 \pm 97$.

Figure 10 shows the results for a series of calculations in which isothermal clouds with a range of temperatures and densities were exposed to the radiation field of the central star. The central star was approximated as a blackbody and the X-ray continua as a series of free-free emitters with the published luminosities and temperatures. The total continuum was attenuated by an effective column density of $10^{22} \mathrm{~cm}^{-2}$ to approximate the extinction of the ionizing radiation by the $\mathrm{H}^{+}$region. Typical planetary nebula abundances and ISM grains were assumed. The clouds had a thickness of $3 \times 10^{15} \mathrm{~cm}$. The figure shows the $1-1 S(7)$ to $0-0 S(7)$ line ratio. The predicted ratio is generally quite small for the values of the $\mathrm{H}_{2}$ density and temperature considered, approaching the upper limit that we identify above only at combinations of low $\mathrm{H}_{2}$ density and temperature.

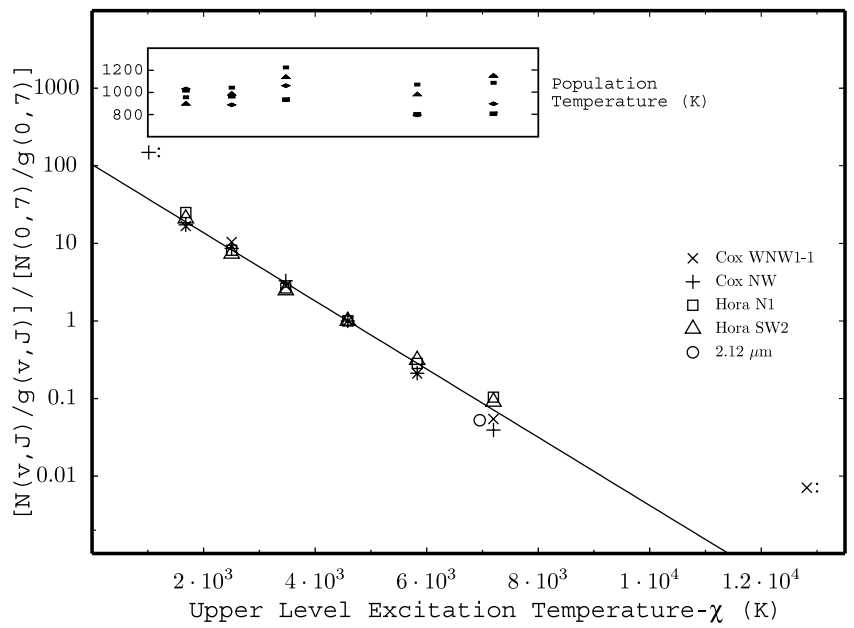

Fig. 9.-Population divided by the statistical weight in various $(v, J)$ states relative to that in the ( $v=0, J=7)$ level [the origin of the $0-0 S(5)$ transition], as a function of the energy of the level expressed in units of the excitation temperature, as described in the text. The line shows the relation if all of the levels were characterized by a gas temperature of $988 \mathrm{~K}$. The inset gives the gas temperature derived for each level, using the $0-0 S(5)$ line for reference, and demonstrates the relatively small dispersion around the average temperature of $988 \mathrm{~K}$. The symbols with colons represent intrinsically uncertain lines, and the circle indicates the position of the $2.12 \mu \mathrm{m}$ line, none of which were used in deriving the best fit.
C98 argued that the total density must exceed $10^{5} \mathrm{~cm}^{-3}$ in order for collisions to dominate and produce the near-singletemperature population distribution. This would be the density to combine with the derived temperature of $900 \mathrm{~K}$ for comparison with the $S(7)$ line ratio, if the emitting zone were predominantly molecular hydrogen. In that case the predicted line ratio is much lower than the upper limit of the observations, and one concludes that the observed weakness of the $0-0 S(7)$ line is not a useful indicator of the excitation mechanism as assumed by H06.

OHF05 derived a density of the molecular hydrogen from observations of the $2.12 \mu \mathrm{m}$ line, finding a value of $6 \times 10^{4} \mathrm{~cm}^{-3}$. However, they assume that the population of the level producing the transition was in LTE at $900 \mathrm{~K}$, which seems to be the case, as the upper state producing the $2.12 \mu \mathrm{m}$ line falls right on the population distribution for this temperature. Using this density would also still indicate that the $S(7)$ line ratio is not a useful

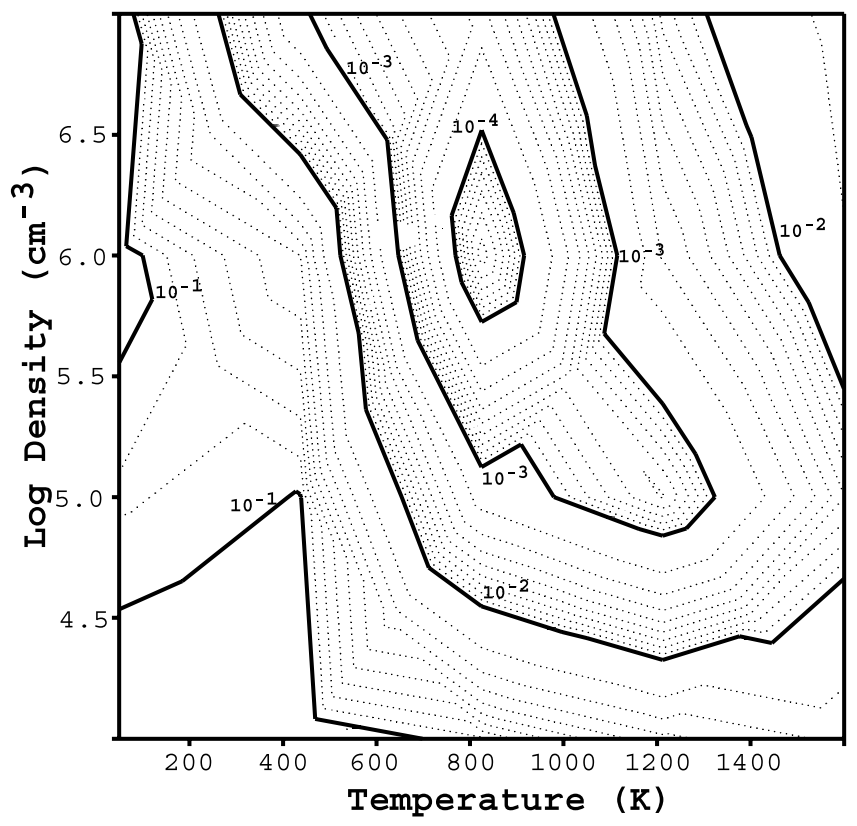

FIG. 10.-Expected flux ratio of the $1-1 S(7)$ and $0-0 S(7)$ lines as a function of the local temperature and density in the PDR. 
indicator of the excitation mechanism. However, the challenge remains of explaining the unexpected combination of temperature and density.

Most of the existing calculations of $\mathrm{H}_{2}$ population distributions and resulting emission spectra have been done for the case of a PDR near an H II region (Black \& van Dishoeck 1987). The stellar radiation field of an O or B star peaks near the wavelengths that excite the electronic transitions of $\mathrm{H}_{2}$, at about $1000 \AA$. The main effect of illumination by this continuum is absorption into excited electronic states that then decay into excited vibrationrotation levels within the ground electronic state of $\mathrm{H}_{2}$. A very nonthermal distribution is produced by these electronic photoexcitations, as $\mathrm{H} 06$ pointed out.

However, the $\mathrm{C} 98$ and $\mathrm{H} 06$ spectra discussed in $\S 4.2$ show that the population distribution within lower levels of $\mathrm{H}_{2}$ is well matched by a thermal distribution at about $900 \mathrm{~K}$. The nonthermal population distribution produced by $\mathrm{O}$ star photoexcitation is simply not seen. This is not surprising, since the environment is so dissimilar from that near a main-sequence $\mathrm{O}$ or $\mathrm{B}$ star. The stellar radiation field of the Helix Nebula central star peaks at much shorter wavelengths than that of an O star, and $\S$ 4.1.2 shows that the observed stellar continuum in the 912-1100 $\AA$ interval is too small to account for the luminosity of the $\mathrm{H}_{2}$ lines by photoexcitation, since the excitation of the fluorescent lines uses but a small fraction of the total energy in the 912-1100 $\AA$ interval. We agree with $\mathrm{H} 06$ that the pure rotational $\mathrm{H}_{2}$ lines are not photonpumped, but not for the reasons they give. They are far too bright to be produced by photoexcitation by the current stellar continuum. We establish in $\S 4$ that the PDRs of the knots almost certainly cannot be powered by shocks. Another energy source is needed.

\subsection{The Nature of the Dissociation Front in the Knots}

In $\S \S 4.1 .1$ and 4.1.2 we examine the energy budget for the Helix Nebula, establishing that only the central star radiation more energetic than $13.6 \mathrm{eV}$ (the extreme-ultraviolet radiation [EUV]) has enough power to drive the large total flux of $\mathrm{H}_{2}$ emission that is observed, thus expanding and quantifying the conclusions in C98. This means that the soft X-ray heating processes and 912-1100 $\AA$ (FUV) photoexcitation mechanisms (Natta \& Hollenbach 1998) do not explain the Helix observations. The absence of a stellar wind and other timescale considerations have already established (OHF05) that shocks cannot be powering the $\mathrm{H}_{2}$ emission, and in $\S 4.3$ we show why the justification of $\mathrm{H06}$ for a shock interpretation is incorrect. Although Phillips (2006) established a loose correlation between soft $\mathrm{X}$-ray flux and $\mathrm{H}_{2}$ emission, this correlation is probably secondary rather than primary, as there is insufficient $\mathrm{X}$-ray emission to power the $\mathrm{H}_{2}$ emission; but, the stars that are strong $\mathrm{H}_{2}$ emitters have high temperatures, such as the central star in the Helix Nebula. This means that these stars share the property of the EUV radiation being dominant over FUV radiation. Clearly, a new process is required. Based on this observational foundation, we identify a new state of equilibrium that may be common but has not previously been identified. A new mechanism using the EUV radiation is briefly described here and will be elaborated on in a future publication.

The ionized flows from the knots are advection dominated, meaning that recombinations are relatively unimportant (Henney 2001). As a result, neither the FUV nor X-ray models (Hollenbach \& Tielens 1997; Natta \& Hollenbach 1998) is relevant to the dissociation fronts in the Helix knots. Instead, the dissociation front merges with the ionization front (Bertoldi \& Draine 1996), and the dissociation of $\mathrm{H}_{2}$ in this merged front is controlled by the ionizing EUV. The fact that neutral hydrogen $21 \mathrm{~cm}$ is not observed in the inner region of the Helix Nebula (Rodríguez et al. 2002) where the optically bright knots are found supports this model, and the appearance of $21 \mathrm{~cm}$ emission from the more distant and fainter outer-ring knots indicates that a neutral hydrogen zone is only present there.

The low-ionization parameter found in the Helix knots leads to substantial deviations from ionization and thermal equilibrium, since the dynamical time is shorter than the ionization and heating times. The effects of this on the emission from the ionized gas were discussed at length in OHF05. The dissociation of $\mathrm{H}_{2}$ in such a front is predominantly due to chemical reactions with ionized species such as $\mathrm{O}^{+}$and is therefore a strong function of the ionization fraction, which is determined by the absorption of EUV radiation. The radiation field is largely determined by the opacity in the fraction of hydrogen that is neutral, the key element being its determination of the amount of $\mathrm{O}^{+}$. It is the reaction of $\mathrm{O}^{+}$with $\mathrm{H}_{2}$ that destroys the $\mathrm{H}_{2}$, rather than the much slower rate of photodissociation of $\mathrm{H}_{2}$. This is essentially a one-way process, with $\mathrm{H}_{2}$ entering the zone from the cold molecular core and being converted directly to $\mathrm{H}^{+}$. This transition zone is heated by the photoionization of neutral hydrogen and can be quite broad, and the preliminary models indicate that it can produce warm $\mathrm{H}_{2}$ column densities of about $10^{19} \mathrm{~cm}^{-2}$, as required by the observations (OHF05). To the best of our knowledge, no models of such EUVdominated dissociation regions have been calculated. We are calculating detailed models of such regions, which will be reported on in a future paper, and restrict ourselves here to this brief description.

It is likely that the same process determines the emission seen from the sheath of the tails in $2.12 \mu \mathrm{m}$. The first-order theory for shadowed columns behind optically thick knots was presented by Cantó et al. (1998). They illustrated that the shadowed regions are illuminated by LyC photons emitted from recombining hydrogen, that this radiation was closer to $13.6 \mathrm{eV}$ than the ionizing stellar radiation, and that the flux density of these diffuse LyC photons was about 0.15 times that of the direct $\mathrm{LyC}$ flux from the central star. These diffuse LyC photons are almost certainly the source of the heating of the $\mathrm{H}_{2}$ in the tails, as the surface brightness in $2.12 \mu \mathrm{m}$ is about $0.23 \pm 0.08$ times that in the directly illuminated bright cusp ( $\S 3.6)$. The implausibility of shocks is also true here, and the shortfall of energy from the FUV radiation is even greater than in the cusps because having a strong diffuse FUV radiation field would demand a large optical depth in dust for the nebula as a whole, which is not indicated by its emissionline spectrum. A separate detailed $\mathrm{H}_{2}$ model is required for the tail because the illuminating FUV will be of lower energy and the density much lower than in the bright cusp.

\subsection{The Absence of Radial Features in the Center of the Helix Nebula}

In a recent paper, M05 presented an analysis of images made in the center of the Helix Nebula in $\mathrm{H} \alpha+[\mathrm{N}$ II] in 1992 (technical details described in Meaburn et al. 1998). They presented a highcontrast rendering of the image (see their Fig. 10) and argued that radial "spokes" can be seen to faintly continue inside the boundary of the "cometary globules" to within about $30^{\prime \prime}$ from the central star.

An arguably superior image of the region is available in the Cerro Tololo Inter-American Observatory $4 \mathrm{~m}$ MOSAIC images made in a similar filter $\left(\mathrm{H} \alpha+\left[\mathrm{N}_{\mathrm{II}}\right]\right)$ and resolution, with a pixel scale $\left(0.26^{\prime \prime}\right.$ pixel $\left.^{-1}\right)$. The individual exposures were $300 \mathrm{~s}$, and in the central region, where the fields of the four different pointings overlap, the effective exposure was $1200 \mathrm{~s}$. A straightforward 


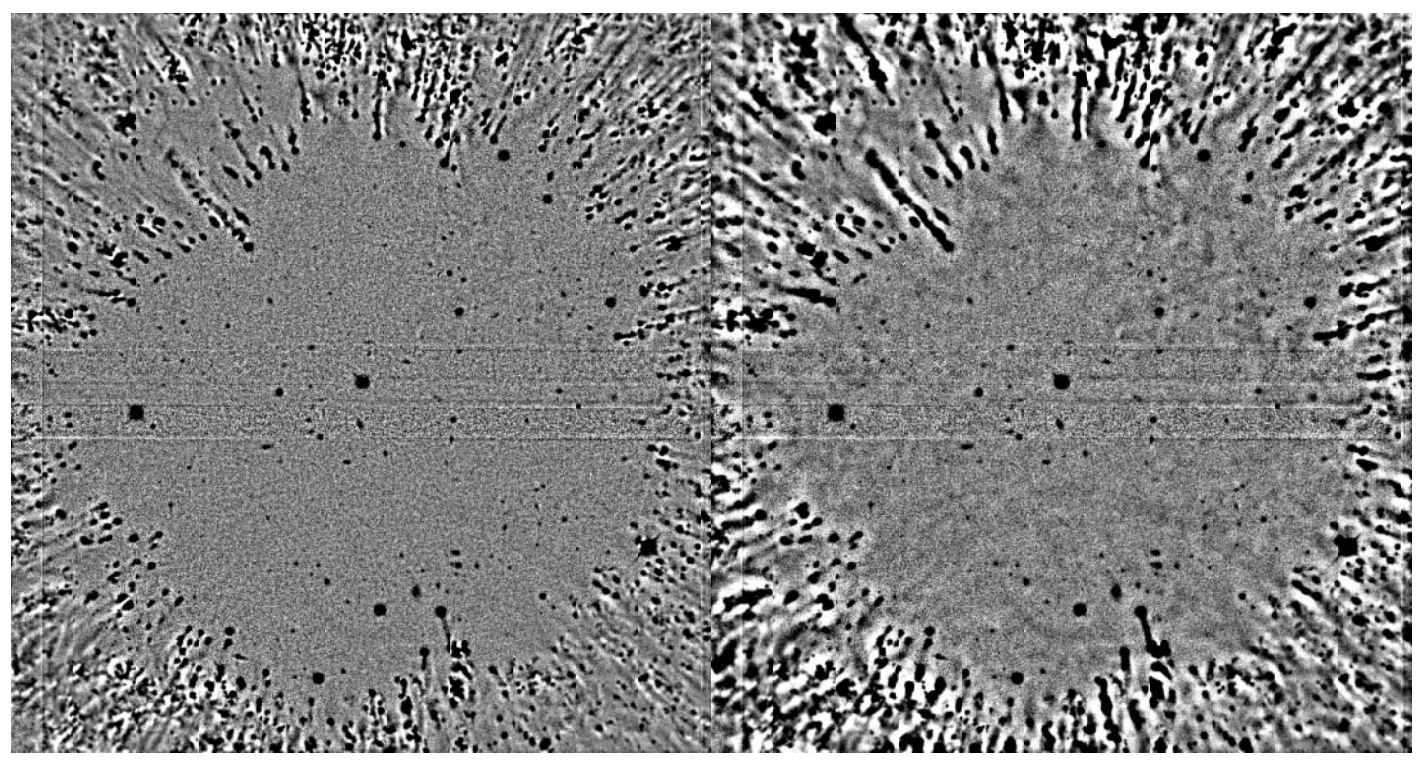

Fig. 11. - $377^{\prime \prime} \times 398^{\prime \prime} \mathrm{H} \alpha+[\mathrm{N} \mathrm{II}]$ image of the center of the Helix Nebula, showing the ratio of the original image divided by a median-filtered image of $20 \times$ 20 pixels (left) and of $40 \times 40$ pixels (right). No inner-region radial spoke features reported by M05 are seen. The horizontal and vertical linear features are the seams of the mosaic formed from several CCDs.

examination of this image using various levels of brightness and contrast did not reveal the features posited by Meaburn et al. (O'Dell 2005). We have now more intensively examined the same images by employing median filters of $20 \times 20$ and $40 \times 40$ pixels and dividing the original images by these, a technique previously employed (OMM04) to enhance the visibility of radial features in the outer parts of the nebula. The results are shown in Figure 11 in negative depiction, or the highly stretched range of intensities of $0.97-1.03$. One sees no indication of radial features extending inside the radius at which the knots disappear. We argue that our images are a better test of such features, since one can see numerous stars and galaxies that do not appear in the M05 image.

This nondetection of such features, even only slightly inside the position of the easily visible bright cusp knots, is a strong argument that this boundary indicates where knots were first formed and does not represent a boundary where knots have been destroyed. An attractive model for generating the initial irregularities that develop into the knots is presented in the calculations of García-Segura et al. (2006), who argue that these should arise at the boundary of shocked material as the initial fast-wind phase of the nebula ends, which is likely to be at about this position within the nebula.

\subsection{A Critique of Stream-Source Models for the Helix Knots}

In a series of papers, Dyson and collaborators have developed a model for the structure of the Helix knots based on the hydrodynamic interaction that results when ionized gas is injected into a subsonic stream that flows past the injection source (Dyson et al. 1993, 2006).

Although the injected ionized gas is assumed to arise from an ionization front on the head of a neutral globule, the radiation transfer and ionization process are not explicitly included in the models. This makes it very difficult to make a meaningful comparison between the results of these models and observations of the Helix Nebula.

Our new $\mathrm{H}_{2}$ observations clearly show ( $\S 3.2$ ) that the limbbrightened edges of the knot tails correspond to an ionization front. In addition, the width of the tail is equal to the width of the bright cusp at the head of the knot and its conic projection (O’Dell 2000). This would seem to conclusively establish that radiation shadowing, rather than hydrodynamic interactions, is the primary determinant of the structure of the tails. In the streamsource model, although tail widths are predicted to be of the same order as the width of the injection source, there is no reason to expect them to be equal unless the parameters are fine-tuned.

The kinematic arguments given in support of the stream-source model (Dyson et al. 2006) also do not stand up to close scrutiny. They are based on the ground-based echelle spectroscopic observations of Meaburn et al. (1998), which seem to show an acceleration of gas along the tail of the knot 427-901. However, a comparison with the much higher resolution HST observations (e.g., ODH $05^{3}$ ). clearly shows that the sample regions used in that observational study all correspond to independent knots that are merely projected onto the tail of 427-901. Therefore, the observed variation in velocity does not represent an acceleration along the tail, but simply a tendency toward higher velocities in knots that are farther from the central star, which has already been noted from CO observations (Young et al. 1999).

\section{CONCLUSIONS}

We have been able to use existing and new observations to reach a number of important conclusions about the knots in the Helix Nebula.

1. There is sufficient energy to power the nebula's $\mathrm{H}_{2}$ emission only in extreme-ultraviolet radiation from the central star with energies $\geq 13.6 \mathrm{eV}$, thus eliminating photoexcitation by the 912-1100 $\AA$ and X-ray flux that has been assumed in previous general models.

2. There is no evidence from infrared emission lines of shock excitation of the knots' $\mathrm{H}_{2}$ emission; the lack of a driving stellar wind and previous arguments of timescale support the same conclusion.

3. Spectrophotometry of multiple lines in four sample regions and the total nebular flux ratio in the $\mathrm{H}_{2} 0-0 S(5)$ and $2.12 \mu \mathrm{m}$ lines indicate that the $\mathrm{H}_{2}$ emitting zones are all about $988 \pm 119 \mathrm{~K}$, closely resembling LTE.

\footnotetext{
${ }^{3}$ See also the image available at http://hubblesite.org/newscenter/archive/ releases/nebula/planetary/2003/11/image/b.
} 
4. The $2.12 \mu \mathrm{m}$ emission from individual knots falls immediately inside the ionized gas zone traced by $\mathrm{H} \alpha$ emission. This is true for both the bright cusps and their fainter tails, the latter establishing that the tails are primarily ionizing radiation shadows, rather than the result of purely hydrodynamic processes.

5. The advection-dominated nature of the knot cusps means that there is no extended neutral hydrogen zone between the cold molecular knot core and the ionized gas layer. This zone of irradiation of $\mathrm{H}_{2}$ by EUV photons is probably the region producing the observed hot gas in the cusps on the star-facing side of the molecular knots and the shadowed regions of the tails.

6. No evidence was found for knots within the He II core, nor were earlier claims verified of linear features extending nearly into the central star, arguing that the knots have only been created outside the high-ionization core.
Anton Koekemoer of the STScI provided valuable guidance in the use of the drizzle package of tasks, making possible the smooth combination of our multiple observations of the same fields. We are grateful to Angela Speck for providing the calibrated $2.12 \mu \mathrm{m}$ image from her Speck et al. (2002) paper and to Pierre Cox for his comments on the $\mathrm{H}_{2}$ physical processes. C. R. O. thanks el Centro de Radioastronomía y Astrofísica, UNAM, Mexico, for generously supporting a 2 week visit in 2006 February, during which initial work for this paper was carried out, and also acknowledges grant GO-10628 from the STScI. G. J. F.'s work was supported in part by grant AR-10653 from the STScI, NASA grant NNG 05GD81G, and NSF grant AST 06-07028. W. J. H. acknowledges financial support from Dirección General de Asuntos del Personal Académico, UNAM, Mexico, project IN112006.
Bertoldi, F., \& Draine, B. T. 1996, ApJ, 458, 222

Black. J. H., \& van Dishoeck, E. F. 1987, ApJ, 322, 412

Bohlin, R. C., Harrington, J. P., \& Stecher, T. P. 1982, ApJ, 252, 635

Burkert, A., \& O’Dell, C. R. 1998, ApJ, 503, 792

Cantó, J, Raga, A., Steffen, W., \& Shapiro, P. R. 1998, ApJ, 502, 695

Cerruti-Sola, M., \& Perinotto, M. 1985, ApJ, 291, 237

Cox, P., et al. 1998, ApJ, 495, L23 (C98)

Dabrowski, I. 1984, Canadian J. Phys., 62, 1639

Dyson, J. E., Hartquist, T. W., \& Biro, S. 1993, MNRAS, 261, 430

Dyson, J. E., Pittard, J. M., Meaburn, J., \& Falle, S. A. E. G. 2006, A\&A, 457, 561

Fazio, G., et al. 2004, ApJS, 154, 10

García-Segura, G., López, J. A., Steffen, W., Meaburn, J., \& Manchado, A. 2006, ApJ, 646, L61

Gonzaga, S., et al. 2005, ACS Instrument Handbook, Ver. 6.0 (Baltimore: STScI), http://www.stsci.edu:8083/hst/acs/documents/handbooks/cycle15/cover.html

Guerrero, M. A., Chu, Y.-H., Gruendl, R. A., Williams, R. M., \& Kaler, J. B. 2001, ApJ, 553, L55

Harris, H. C., et al. 2007, AJ, 133, 631

Henney, W. J. 2001, RevMexAA, 10, 57

Hollenbach, D. J., \& Tielens, A. G. G. M. 1997, ARA\&A, 35, 179

Holtzman, J. A., Burrows, C. J., Castertano. S., Hester, J. J., Trauger, J. T., Watson, A. M., \& Worthey, G. 1995, PASP, 107, 1065

Hora, J. L., Latter, W. B., Smith, H. A., \& Marengo, M. 2006, ApJ, 652, 426 (H06)

Houck, J. R., et al. 2004, ApJS, 154, 18

Huggins, P. J., Forveille, T., Bachiller, R., Cox, P., Ageorges, N., \& Walsh, J. R. 2002, ApJ, 573, L55

Leahy, D. A., Zhang, C. Y., \& Kwok, S. 1994, ApJ, 422, 205

Leahy, D. A., Zhang, C. Y., Volk, K., \& Kwok, S. 1996, ApJ, 466, 352

López-Martín, L., Raga, A. C., Melleman, G., Henney, W. J., \& Cantó, J. 2001, ApJ, 548, 288

Meaburn, J., Boumis, P., López, J. A., Harman, D. J., Bryce, M., Redman, M. P., \& Mavromatakis, F. 2005, MNRAS, 360, 963 (M05)

Meaburn, J., Clayton, C. A., Bryce, M., Walsh, J. R., Holloway, A. J., \& Steffen, W. 1998, MNRAS, 294, 201

Meaburn, J., Walsh, J. R., Clegg, R. E. S., Walton, N. A., \& Taylor, D. 1992, MNRAS, 255, 177

\section{REFERENCES}

Meixner, M., McCullough, P., Hartman, J., O’Dell, C. R., \& Speck, A. K. 2004, in ASP Conf. Ser. 313, Asymmetrical Planetary Nebulae III, ed. M. Meixner et al. (San Francisco: ASP), 234

Meixner, M., McCullough, P. R., Hartman, J., Son, M., \& Speck, A. K. 2005, AJ, 130, 1784 (MX05)

Mellema, G., Raga, A. C., Cantó, J., Lundqvist, P., Balick, B., Steffen, W., \& Noriega-Crespo, A. 1998, A\&A, 331, 335

Natta, A., \& Hollenbach, D. 1998, A\&A, 337, 517

O’Dell, C. R. 1998, AJ, 116, 1346

. 2000, AJ, 119, 2311

2004, PASP, 116, 729

2005, RevMexAA, 23, 5

O’Dell, C. R., Balick, B., Hajian, A. R., \& Henney, W. J. 2002, AJ, 123, 3329

O'Dell, C. R., \& Burkert, A. 1997, in IAU Symp. 180, Planetary Nebulae, ed.

H. J. Habing \& H. J. G. L. M. Lamers (Dordrecht: Kluwer), 332

O’Dell, C. R., \& Doi, T. 1999, PASP, 111, 1316

O’Dell, C. R., \& Handron, K. D. 1996, AJ, 111, 1630

O'Dell, C. R., Henney, W. J., \& Burkert, A. 2000, AJ, 119, 2910 (OHB00)

O'Dell, C. R., Henney, W. J., \& Ferland, G. J. 2005, AJ, 130, 172 (OHF05)

O'Dell, C. R., McCullough, P. R., \& Meixner, M. 2004, AJ, 128, 2339 (OMM04)

Phillips, J. P. 2006, MNRAS, 368, 819

Rauch, T. 2003, A\&A, 403, 709

Rodríguez, L. F., Goss, W. M., \& Williams, R. 2002, ApJ, 574, 179

Shaw, G., Ferland, G. J., Abel, N. P., Stancil, P. C., \& van Hoof, P. A. M. 2005 , ApJ, 624, 794

Speck, A. K., Meixner, M., Fong, D., McCulough, P. R., Moser, D. E., \& Ueta, T. 2002, AJ, 123, 346

Thompson, R. I., Rieke, M., Schneider, G., Hines, D. C., \& Corbin, M. R. 1998, ApJ, 492, L95

Walsh, J. R., \& Ageorges, N. 2003, in IAU Symp. 209, Planetary Nebulae: Their Evolution and Role in the Universe, ed. S. Kwok, M. Dopita, \& R. Sutherland (San Francisco: ASP), 275

Wolniewicz, L., Simbotin, I., \& Dalgarno, A. 1998, ApJS, 115, 293

Wood, K., Mathis, J. S., \& Ercolano, B. 2004, MNRAS, 348, 1337

Young, K., Cox, P., Huggins, P. J., Forveille, T., \& Bachiller, R. 1999, ApJ, 522,387 